\title{
SHAPE DIFFERENTIABILITY OF DRAG FUNCTIONAL AND BOUNDARY VALUE PROBLEM SOLUTIONS FOR FLUID MIXTURE EQUATIONS
}

\author{
Nikolay A. Kucher, Aleksandra A. Zhalnina* \\ Kemerovo State University, \\ Krasnaya Str. 6, Kemerovo, 650043 Russian Federation \\ *e-mail: qwert1776@yandex.ru
}

Received April 18, 2016;

Accepted in revised form May 24, 2016;

Published December 30, 2016

\begin{abstract}
Problems of optimal design of various elements of technical structures stimulate mathematical statements of new problems of continuum mechanics and hydrodynamics in particular. This study refers to problems of shape optimization of profiles in a flow of fluid or gas. The paper deals with properties of solutions and their functional of inhomogeneous boundary value problem for nonlinear composite type partial differential equation system, simulating the a mixture of viscous compressible fluids (gases) flowing around an obstacle. Methods of the theory of partial differential equations, functional analysis and, in particular, the results on the solvability of boundary value problems for transport and Stokes equations established the well-posedness of a linear boundary value problem with singular coefficients (the problem of the original problem solution difference). This result allowed to obtain the uniqueness theorem to determine the nature of solutions dependence on the shape of the flow range and to prove domain differentiability of the solutions considered. Domain differentiability of the solution functional reflecting the force of the obstacle resistance to the incident flow is proved. A formula to equate this derivative as a sum of two summands, one of which clearly depends on mapping setting the domain shape, and the other can be expressed in terms of the so-called adjoint state, depending only on the solution of the original problem in a non-deformed domain. The functional derivative formulas may be used as the basis for building a numerical algorithm for finding the optimal shape of the body in a flow of mixture of viscous compressible fluids.
\end{abstract}

Keywords: boundary value problem, mixture of viscous compressible fluids, conjugate problem, flowing around an obstacle, material derivative, shape derivative

DOI: $10.21603 / 2500-1418-2016-1-2-41-56$.

\section{INTRODUCTION}

Search for the optimal shape (with the lowest drag) of the obstacle in a flow of a mixture of viscous compressible fluids (gases) is eventually associated with the problem of derivation of the shape functional, expressing the force of the obstacle resistance to the incident flow. This problem, in turn, requires a study of well-posedness of inhomogeneous boundary value problem for the corresponding equations and study of dependence of this boundary value problem solutions on the flow region shape.

Most of the known results for Navier-Stokes equations for viscous compressible fluids and moreover for equations of mixtures of such media concerns flows in are as bounded by impenetrable walls, while the results of study of inhomogeneous boundary value problems remain fairly modest. Among the papers dealing with the last issue, we'd like to mention [1], which proves existence theorem for non-stationary Navier-Stokes equations for viscous compressible fluid with constant boundary value conditions, and [2], which establishes existence of a weak solution of barotropic viscous gas flow equations in convex domains with the outlet, independent on the time variable. Local strong solutions (close to a uniform flow) of stationary problems with inhomogeneous boundary value conditions were studied in [3-5] for two-dimensional domains on the hypothesis that the velocity field at the boundary of the flow region is close to a prescribed constant. Important results relating to the existence of strong solutions of inhomogeneous boundary value problems for stationary Navier-Stokes equations in case of small Reynolds and Mach numbers were obtained in [6-8]. Results on the well-posedness of an inhomogeneous boundary value problem for the equations of mixtures of viscous compressible fluids were obtained in [9].

The shape optimization theory is a section of variational calculus, where the functional arguments are shapes of geometrical and physical objects. A classic example of the shape optimization problem is the isoparametric Newto n's problem of the body of least resistance. Description of the general theory and bibliography on this subject are available in [10-15]. The first global result concerning dependence on the solution region of compressible Navier-Stokes equations was obtained by Feireisl [16], and was further developed

Please cite this article inpress as: Kucher N.A., Zhalnina A.A. Shape differentiability of drag functional and boundary value problem solutions for fluid mixture equations. Science Evolution, 2016, vol. 1, no. 2, pp. 41-56. doi: 10.21603/2500-4239-2016-1-2-41-56.

Copyright (C) 2016, KemSU. This is an open access article distributed under the terms of the Creative Commons Attribution 4.0 International License (http:// creativecommons.org/licenses/by/4.0/), allowing third parties to copy and redistribute the material in any medium or format and to remix, transform, and build upon the material for any purpose, even commercially, provided the original work is properly cited and states its license. This article is published with open access at http:// science-evolution.ru/ 
in a series of papers of Plotnikov, Ruban, Sokolowski $[7,8,17-20]$. These studies also provide an algorithm for calculation of the drag functional derivatives determined in the collection of domains.

The problem statement is as follows. Range of flow of viscous compressible fluid mixture is a domain $\Omega=B \backslash S$ of Euclidean space $\mathbb{R}^{3}$ of points $x=\left(x_{1}, x_{2}, x_{3}\right)$, external with respect to an obstacle $S$ (which is assumed to be a compact set) and bounded by a closed surface $\Sigma$. Let us assume that $x \mapsto \vec{T}(x)$ denotes the vector field of class $C^{2}\left(\mathbb{R}^{3}\right)$, equal to zero in the neighborhood of the boundary $\Sigma$. Let us define a mapping $x \mapsto y=\vec{T}_{\varepsilon}(x)=x+\varepsilon \vec{T}(x)$ which defines the perturbation of the obstacle $S$. For small $\varepsilon$, mapping $x \mapsto \vec{T}_{\varepsilon}(x)$ is a diffeomorphism of the flow region $\Omega$ onto $\Omega_{\varepsilon}=B \backslash S_{\varepsilon}$, where $S_{\varepsilon}=\vec{T}_{\varepsilon}(S)$ is perturbed obstacle in a flow.

Stationary motion of the mixture of viscous compressible fluids in the region $\Omega_{\varepsilon}$ is described by the following equations [21]:

$$
\begin{gathered}
\sum_{j=1}^{2} L_{i j}\left(\vec{u}_{\varepsilon}^{(j)}\right)+\operatorname{Re} \rho_{i \varepsilon}\left(\vec{u}_{\varepsilon}^{(i)} \cdot \nabla\right) \vec{u}_{\varepsilon}^{(i)}+\frac{\mathrm{R} e}{\mathrm{M} a^{2}} \nabla p_{i \varepsilon}\left(\rho_{i \varepsilon}\right)+\vec{J}^{(i)}=0 \text { in } \Omega_{\varepsilon}, i=1,2 \\
\operatorname{div}\left(\rho_{i \varepsilon} \vec{u}_{\varepsilon}^{(i)}\right)=0 \text { in } \Omega_{\varepsilon}, i=1,2
\end{gathered}
$$

where $\vec{u}_{\varepsilon}^{(1)}, \vec{u}_{\varepsilon}^{(2)}$ represent the velocity fields of the mixture components; $\rho_{1 \varepsilon}, \rho_{2 \varepsilon}$ are the component density functions, and corresponding pressures $p_{i \varepsilon}=p_{i \varepsilon}\left(\rho_{i \varepsilon}\right), i=1,2$, are assumed to be sufficiently smooth functionsoftheirdensities; $\mathrm{R} e$ and $\mathrm{M} a$ denotethe
Reynoldsand Mach numbers, respectively; $L_{i j}, i, j=1,2$, refers to the second-order differential operators $L_{i j}\left(\vec{u}^{(j)}\right)=-\mu_{i j} \Delta \vec{u}^{(j)}-\left(\mu_{i j}+\lambda_{i j}\right) \nabla \mathrm{d} \dot{v} \vec{u}^{(j)}, i, j=1,2$, where constant (dimensionless) viscosity coefficients $\mu_{i j}, \lambda_{i j}$ satisfy the conditions

$$
\begin{gathered}
\mu_{11}>0,4 \mu_{11} \cdot \mu_{22}-\left(\mu_{12}+\mu_{21}\right)^{2}>0, \lambda_{11}+2 \mu_{11}>0 \\
4\left(\lambda_{11}+2 \mu_{11}\right)\left(\lambda_{22}+2 \mu_{22}\right)-\left(\lambda_{12}+2 \mu_{12}+\lambda_{21}+2 \mu_{21}\right)^{2}>0 .
\end{gathered}
$$

Summands $\vec{J}^{(i)}=(-) 1^{(i)} a\left(\vec{u}_{\varepsilon}^{(2)}-\vec{u}_{\varepsilon}^{(1)}\right), a=$ const $>0, i=1,2$, describe intensity of the momentum exchange between the mixture components [22,23]. Equations (1) and (2) represent the laws of conservation of momentum and mass of the mixture components, respectively.

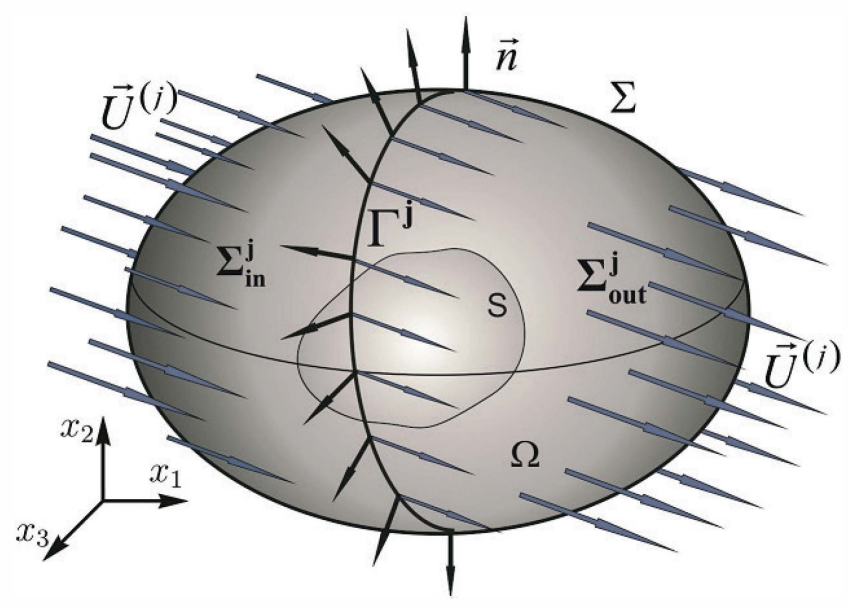

(a)

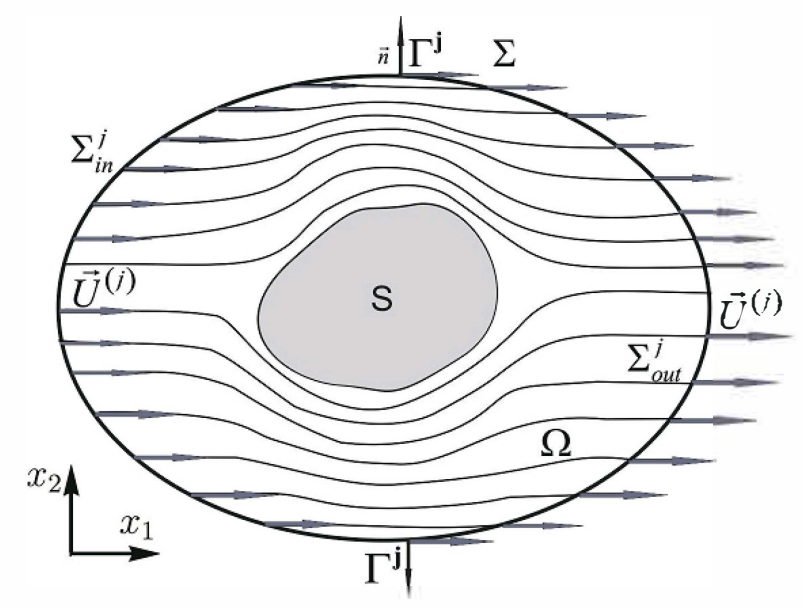

(b)

Fig. 1. Diagrams of the flow of the jth component of the mixture around the obstacle: (a) three-dimensional flow; (b) plane section.

For statement of boundary value conditions let us use the vector fields $\vec{U}^{(j)}, j=1,2$, of class $C^{3}\left(\mathbb{R}^{3}\right)$, vanishing in a neighborhood of the set $S$. Let us use vector functions $\vec{U}^{(j)}$ on the boundary $\Sigma$ of the domain $B$ to allocate "inflow" areas: $\Sigma_{\text {in }}^{j}=\left\{x \in \Sigma: \vec{U}^{(j)} \cdot \vec{n}<0\right\}, j=1,2$, and "outflow" areas: $\Sigma_{\text {out }}^{j}=\left\{x \in \Sigma: \vec{U}^{(j)} \cdot \vec{n}>0\right\}, j=1,2$ (see Fig. 1). Let us assume that the following conditions are satisfied.

Condition 1. Sets $\Gamma^{j}=c l \Sigma_{i n}^{j} \cap\left(\Sigma \backslash \Sigma_{i n}^{j}\right), j=1,2$, ("characteristic" surface areas) are closed one-dimensional varifolds, such that $\Sigma=\Sigma_{\text {in }}^{j} \cup \Gamma^{j} \cup \Sigma_{\text {out }}^{j}$, and, among other things: $\int_{\Sigma} \vec{U}^{(j)} \cdot \vec{n} s d=0, j=1,2 ; \vec{U}^{(j)} \cdot \nabla\left(\vec{U}^{(j)} \cdot \vec{n}\right)>C>0$ on $\Gamma^{j}, j=1,2$,
where $C>0$ is a constant.

Adjoin the following boundary value conditions to equations (1), (2)

$$
\vec{u}_{\varepsilon}^{(j)}=\vec{U}^{(j)} \text { on } \Sigma, \quad \vec{u}_{\varepsilon}^{(j)}=0 \text { on } \partial S_{\varepsilon}, \quad \rho_{j \varepsilon}=\rho_{j \varepsilon}^{0} \text { on } \Sigma_{i n}^{j}, j=1,2
$$

where $\rho_{j \varepsilon}^{0}, j=1,2$, are prescribed positive constants. 
The force of drag to incident flow from the obstacle $S_{\varepsilon}$ is expressed by the formula

$$
J_{D}\left(S_{\varepsilon}\right)=-\vec{U}^{\infty} \sum_{i=1}^{2} \int_{\partial S_{\varepsilon}}\left(\sum_{j=1}^{2}\left[\mu_{i j}\left(\nabla \vec{u}_{\varepsilon}^{(\mathrm{j})}+\left(\nabla \vec{u}_{\varepsilon}^{(\mathrm{j})}\right)^{T}\right)+\lambda_{i j} \operatorname{div} \vec{u}_{\varepsilon}^{(\mathrm{j})} \mathbf{I}\right]-\frac{R e}{M a^{2}} p_{i}\left(\rho_{\varepsilon}\right) \mathbf{I}\right) \cdot \vec{n} d s
$$

where $\vec{U}^{\infty}$ is the constant vector that simulates the flow rate at "infinity". The problem of minimization this functional is solution to the problem of selection the optimal shape of the obstacle.

The problem (1)-(3) can be conveniently reduced to a boundary value problem in the unperturbed domain $\Omega$ for one-parameter family of differential equations with perturbed coefficients. For this purpose, let us introduce the functions $\vec{u}^{(i)}$ and $\rho_{i}, i=1,2$ defined in $\Omega$ in accordance with the following formulas:

$$
\vec{u}^{(i)}(x)=N(x) \vec{u}_{\varepsilon}^{(i)}(x+\varepsilon \vec{T}(x)), \rho_{i}(x)=\rho_{i \varepsilon}(x+\varepsilon \vec{T}(x)), x \in \Omega, i=1,2,
$$

where $\mathbf{N}(x)=\left(\operatorname{det} M(x) M^{-1}(x), M(x)=I+\varepsilon D \vec{T}(x), D \vec{T}(x)=\left\{\frac{\partial T_{i}(x)}{\partial x_{j}}\right\}\right.$ is the Jacobi matrix of the mapping $x \mapsto \vec{T}(x)$.

As a result of this transformation, the problem (1)-(3) is transformed into the problem

$$
\begin{gathered}
\sum_{j=1}^{2} \mu_{i j} \Delta \vec{u}^{(j)}-\nabla q_{i}=\sum_{j=1}^{2} \mu_{i j} \mathcal{A}\left(\vec{u}^{(j)} ; N\right)+\operatorname{Re} \mathcal{B}\left(\rho_{i}, \vec{u}^{(i)}, \vec{u}^{(i)} ; N\right)+(-1)^{i} \mathcal{S}\left(\vec{u}^{(2)}-\vec{u}^{(1)} ; N\right) \text { in } \Omega \\
\operatorname{div} \vec{u}^{(i)}=\sum_{j=1}^{2} g \sigma_{i j} p_{j}-\sum_{j=1}^{2} g \gamma_{i j} q_{j} \text { in } \Omega \\
\vec{u}^{(i)} \cdot \nabla \rho_{i}+\rho_{i} \sum_{j=1}^{2} g \sigma_{i j} p_{j}=\rho_{i} \sum_{j=1}^{2} g \gamma_{i j} q_{j} \text { in } \Omega, \\
\vec{u}^{(i)}=\vec{U}^{(i)} \text { on } \Sigma, \vec{u}^{(i)}=0 \text { on } \partial S, \rho_{i}=\rho_{i}^{0} \text { on } \Sigma_{i n}^{i}, i=1,2 .
\end{gathered}
$$

Where $g=g(x ; N)=\sqrt{\mathrm{d} t e \mathbf{N}(x)}$; linear operators $\mathcal{A}, \mathcal{S}$ and non-linear mapping $\mathcal{B}$ are defined according to the formulas

$$
\begin{gathered}
\mathcal{A}(\vec{u} ; \mathbf{N})=\Delta \vec{u}-\left(\mathbf{N}^{T}\right)^{-1} \operatorname{div}\left(g^{-1} \mathbf{N N}^{T} \nabla\left(\mathbf{N}^{-1} \vec{u}\right)\right), \\
\mathcal{B}(\rho, \vec{u}, \vec{w} ; \mathbf{N})=\rho\left(\mathbf{N}^{T}\right)^{-1}\left(\vec{u} \nabla\left(\mathbf{N}^{-1} \vec{w}\right)\right), \quad \mathcal{S}(\vec{u} ; \mathbf{N})=g \cdot a\left(\mathbf{N}^{T}\right)^{-1} \mathbf{N}^{-1} \vec{u} ;
\end{gathered}
$$

$q_{i}=-\sum_{j=1}^{2} g^{-1}\left(\mu_{i j}+\lambda_{i j}\right) \mathrm{d} i v \vec{u}^{(j)}+\frac{\mathrm{R} e}{\mathrm{Ma}^{2}} p_{i}\left(\rho_{i}\right), i=1,2$, are effective viscous pressures; $\gamma_{i j}$ are elements of the matrix $\varkappa^{-1}$, inverse of the matrix $\varkappa$ which elements are $\mu_{i j}+\lambda_{i j}, i, j=1,2 ; \sigma_{i j}=\frac{\mathrm{R} e}{\mathrm{M}^{2}} \gamma_{i j}$.

The solution to (5) is built in the form of disturbance of specially selected sufficiently smooth flow $\vec{u}_{*}^{(i)}, \rho_{i}^{*}, q_{i}^{(*)}$, i. e.

$$
\vec{u}^{(i)}=\vec{u}_{*}^{(i)}+\vec{v}^{(i)}, \rho_{i}=\rho_{i}^{*}+\varphi_{i}, q_{i}=q_{i}^{*}+\pi_{i}+\Lambda \cdot p_{i}\left(\rho_{i}^{*}\right)+\sum_{j=1}^{2}\left(\mu_{i j}+\lambda_{i j}\right) m_{j}
$$

where $\rho_{i}^{*}=\rho_{i}^{0}=$ const, $\Lambda=\frac{\mathrm{R} e}{\mathrm{M} a^{2}}, m_{j}$ are constants serving for control the mass of the mixture components in a domain $\Omega$. As a result, a problem for perturbations [9] becomes the main object of study:

$$
\begin{gathered}
\sum_{j=1}^{2} \mu_{i j} \Delta \vec{v}^{(j)}-\nabla \pi_{i}=\sum_{j=1}^{2} \mu_{i j} \mathcal{A}\left(\vec{u}^{(j)} ; \mathbf{N}\right)+\operatorname{Re} \mathcal{B}\left(\rho_{i}, \vec{u}^{(i)}, \vec{u}^{(i)} ; \mathbf{N}\right)+(-1)^{i} \mathcal{S}\left(\vec{u}^{(2)}-\vec{u}^{(1)} ; \mathbf{N}\right) \text { in } \Omega, \\
\operatorname{div} \vec{v}^{(i)}=g \sum_{j=1}^{2} \frac{1}{\rho_{i}^{*}} \tau_{i j} \varphi_{j}-g \Phi_{i}[\vec{\theta}]-g m_{i} \text { in } \Omega, \\
\vec{u}^{(i)} \cdot \nabla \varphi_{i}+\tau_{i i} \varphi_{i}=\Psi_{i}[\vec{\theta}]+g m_{i} \rho_{i} \text { in } \Omega, \\
\vec{v}^{(i)}=0 \text { on } \partial \Omega, \quad \varphi_{i}=0 \text { on } \sum_{i n}^{i}, \quad \Pi \pi_{i}=\pi_{i}\left(\Pi q=q-\frac{1}{|\Omega|} \int_{\Omega} g d x\right), i=1,2 ; \\
\vec{m}=\left(m_{1}, m_{2}\right)^{T}, \vec{m}=(k I-A)^{-1} \vec{f}, A=\left\{a_{i j}\right\}_{i, j=1}^{2}, \vec{f}=\left(f_{1}, f_{2}\right)^{T}, \\
k=\int_{\Omega} g d x, a_{i j}=\frac{1}{\rho_{i}^{*}} \int_{\Omega} g \cdot \rho_{j} \cdot \zeta_{i}^{(j)} d x, f_{i}=\frac{1}{\rho_{i}^{*}} \int_{\Omega}\left(\sum_{j=1}^{2} \zeta_{i}^{(j)} \cdot \Psi_{j}[\vec{\theta}]-g \Phi_{i}[\vec{\theta}]\right) d x ; \\
-\operatorname{div}\left(\vec{u}^{(i)} \cdot \zeta_{j}^{(i)}\right)+\tau_{i i} \cdot \zeta_{j}^{(i)}=\tau_{j l} g \text { in } \Omega_{j}, \zeta_{j}^{(i)}=0 \text { on } \Sigma_{\text {out }}^{(i)}, i, j=1,2 .
\end{gathered}
$$

Here, the constant parameters $\tau_{i j}$ are related in a known manner with the viscosity coefficients 
$\mu_{i j}, \lambda_{i j}$ and prescribed boundary values $\rho_{i}^{0} ; \quad \Phi_{i}[\vec{\theta}], \Psi_{i}[\vec{\theta}] \quad$ are the known functions of the vector $\vec{\theta}=\left(\vec{v}^{(1)}, \vec{v}^{(2)} ; \pi_{1}, \pi_{2}, \varphi_{1}, \varphi_{2}\right)$ components, expressions of which are given in [9], where the existence theorem is proved as well.

Theorem 1. Let the surface $\Sigma$ and vector fields $\vec{U}^{(1)}, \vec{U}^{(2)}$ satisfy the condition 1. Then there are such numbers $\sigma^{*}>1$ and $\tau^{*} \in(0,1)$ that if the matrix $\mathbf{N}$ is selected based on the condition $\|I-\mathbf{N}\|_{\tilde{N}^{2}(\Omega)} \leq \tau^{2}, \tau \in\left(0, \tau^{*}\right], p_{j}\left(\rho_{j}\right) \in C^{3}(0, \infty), j=1,2$, and the problem parameters are such that $\frac{1}{\Lambda} \leq \tau^{2}$, $\operatorname{Re} \leq \tau^{2}, a \leq \tau^{2},\left|\tau_{12}\right| \leq \tau,\left|\tau_{21}\right| \leq \tau, \quad \tau_{i i} \geq \sigma^{*}, i=1,2$, $\tau \in\left(0, \tau^{*}\right]$, then the problem (6) has a solution $\vec{\theta}=\left(\vec{v}^{(1)}, \vec{v}^{(2)} ; \pi_{1}, \pi_{2}, \varphi_{1}, \varphi_{2}\right), m_{i}, \zeta_{i}^{(j)}, i, j=1,2$,

$$
\|u\|_{W^{s, r}(\Omega)}=\|u\|_{L^{r}(\Omega)}+|u|_{s, r, \Omega},|u|_{s, r, \Omega}=\int_{\Omega \times \Omega}|x-y|^{-3}\left(\frac{|u(x)-u(y)|}{|x-y|^{s}}\right)^{r} d x d y .
$$

Ingeneral, thespace $W^{l+s, r}(\Omega), 0<s<1,1 \leq r<\infty, l \geq 0$ is an integer and is defined as the space of measurable functions with a finite norm.

$$
\|u\|_{W^{l+s, r}(\Omega)}=\|u\|_{W^{l, r}(\Omega)}+\sup _{\mid \alpha \models l}\left\|D^{\alpha} u\right\|_{W^{s, r}(\Omega)} .
$$

$W_{0}^{s, r}(\Omega), \quad 0 \leq s \leq 1$ refers to a closed subspace $W^{s, r}\left(\mathbb{R}^{3}\right)$ consisting of all functions $u \in W^{s, r}\left(\mathbb{R}^{3}\right)$ vanishing outside the domain $\Omega$. For the purpose of $0<s<1 \quad 1<r<\infty$, let $\mathcal{W}_{0}^{s, r}(\Omega)$ denote the interpolation space $\left[W_{0}^{0, r}(\Omega) W_{0}^{1, r}(\Omega)\right]_{s, r}$ with the norm determined by the real interpolation.

Let us assume that $0 \leq s \leq 1,1<r<\infty, \frac{1}{r}+\frac{1}{r^{\prime}}=1$. Let $\mathcal{W}^{-s, r}(\Omega)$ denote completion of $L^{r}(\Omega)$ in the norm

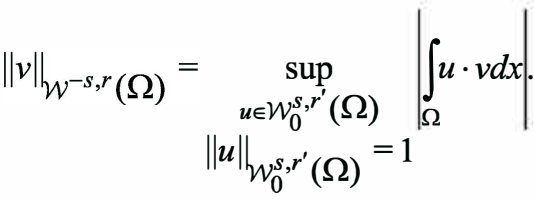

$$
\begin{aligned}
& \mathcal{U}^{s, r}=\mathcal{W}^{s-1} r(\Omega) \times W^{s, r}(\Omega) \times \mathbb{R}, \mathcal{V}^{s, r}=W^{s+1} r(\Omega) \times W^{s, r}(\Omega) \times \mathbb{R}, \\
& Z^{s, r}=\mathcal{W}^{s-\downarrow} r(\Omega) \cap L^{2}(\Omega) \\
& \mathcal{E}^{s, r}=Z^{s, r} \times X^{s, r} \times \mathbb{R}, \mathcal{F}^{s, r}=V^{s, r} \times X^{s, r} \times \mathbb{R},
\end{aligned}
$$

Membership of the vector magnitude $\vec{F}$ of the direct product of spaces $W_{1} \times W_{2} \times W_{3}$ is to be understood in the sense that $\vec{F}$ is made up of three components (scalar or vector), separated by semicolon $\vec{F}=\left(F_{1} ; F_{2} ; F_{3}\right)$ and at the same time $F_{1} \in W_{1}, F_{2} \in W_{2}, F_{3} \in W_{3}$. If $W_{1}=W_{2}=W_{3}=W$, write $\vec{F} \in W$ and separate the vector components by comma.

\section{DOMAIN DEPENDENCE OF SOLUTIONS}

The most important stage of the shape optimization study is to prove the uniqueness of solution of the
As is known from [7], space $\mathcal{W}^{-s, r}(\Omega)$ for $0<s<1$, $1<r<\infty$ is topologically and algebraically isomorphic to Banach space $\left(\mathcal{W}_{0}^{s, r^{\prime}}(\Omega)\right)^{\prime}$, dual to $\mathcal{W}_{0}^{s, r^{\prime}}(\Omega)$, and can be identified with it.

For any $0 \leq s \leq 1,1<r<\infty$, Banach space $\mathbb{W}^{-s, r}(\Omega)$ is completion of $L^{r}(\Omega)$ in the norm.

$$
\|v\|_{\mathbb{W}^{-s, r}(\Omega)}=\left\|\mathcal{L}_{v}\right\|_{\left(W^{s, r^{\prime}}(\Omega)\right)^{\prime}},
$$

where $\mathcal{L}_{v}(u)=\langle v, u\rangle=\int_{\Omega} v(x) \cdot u(x) d x$ is a continuous functional in the space $W^{s, r^{\prime}}(\Omega)$, continuously embedded in $L^{r^{\prime}}(\Omega)$. As is known [7] that if $\Omega$ is a bounded domain in $\mathbb{R}^{3}$ with a boundary of class $C^{1}$, then $\mathbb{W}^{-s, r}(\Omega)$ is algebraically and topologically isomorphic to the dual space $\left(W^{s, r^{\prime}}(\Omega)\right)^{\prime}$, and can be identified with it. In addition, let us introduce functional

problem (6) and differentiability of its solution with reference to the parameter $\varepsilon$. For this purpose it is necessary to study dependence of these solutions on the matrix $\mathbf{N}$ completely determined by the flow range deformation.

At this stage, the matrix $\mathbf{N}$ structure is irrelevant and therefore the results obtained below are valid for an arbitrary smooth matrix-valued function $\mathbf{N}(x), x \in \Omega$.

1. The problem for differences. For difference $\vec{q}_{0}-\vec{q}_{1}$,

$$
\vec{q}_{i}=\left(\vec{v}_{i}^{(1)}, \vec{v}_{i}^{(2)} ; \pi_{1}^{i}, \pi_{2}^{i}, \varphi_{1}^{i}, \varphi_{2}^{i}, \zeta_{1 i}^{(1)}, \zeta_{2 i}^{(1)}, \zeta_{1 i}^{(2)}, \zeta_{2 i}^{(2)} ; m_{1}^{i}, m_{2}^{i}\right), i=0,1,
$$


of solutions to the problem (6) obtained according to Theorem 1 and corresponding to different matrices $\mathbf{N}_{0}$ and $\mathbf{N}_{1}$, let us introduce the following notations

$$
\vec{w}^{(j)}=\vec{v}_{0}^{(j)}-\vec{v}_{1}^{(j)}, \omega_{j}=\pi_{j}^{0}-\pi_{j}^{1}, \psi_{j}=\varphi_{j}^{0}-\varphi_{j}^{1}, n_{j}=m_{j}^{0}-m_{j}^{1}, \xi_{j}^{(k)}=\zeta_{j 0}^{(k)}-\zeta_{j 1}^{(k)}, k, j=1,2 .
$$

From (6), it follows that the vector function

$$
\vec{q}_{0}-\vec{q}_{1}=\left(\vec{w}^{(1)}, \vec{w}^{(2)} ; \omega_{1}, \omega_{2}, \psi_{1}, \psi_{2}, \xi_{1}^{(1)}, \xi_{2}^{(1)}, \xi_{1}^{(2)}, \xi_{2}^{(2)} ; n_{1}, n_{2}\right)
$$

is the solution to the following linear problem

$$
\begin{gathered}
\sum_{j=1}^{2} \mu_{i j} \Delta \vec{w}^{(j)}-\nabla \omega_{i}=\sum_{j=1}^{2} \mu_{i j} \mathcal{A}_{0}\left(\vec{w}^{(j)}\right)+\operatorname{Re} \mathcal{L}\left(\psi_{i}, \vec{w}^{(i)}\right)+\mathcal{D}_{i}+(-) 1^{i}\left(\mathcal{E}+\mathcal{S}_{0}\left(\vec{w}^{(2)}-\vec{w}^{(1)}\right)\right. \\
\operatorname{div} \vec{w}^{(i)}=\sum_{j=1}^{2} \alpha_{i j} \psi_{j}+\sum_{j=1}^{2} \beta_{i j} \omega_{j}+\gamma_{i} n_{i}+\delta_{i} d, \\
\vec{u}_{0}^{(i)} \cdot \nabla \psi_{i}+\tau_{i} \psi_{i}=-\vec{w}^{(i)} \cdot \nabla \varphi_{i}^{1}+\sum_{j=1}^{2} \hat{\alpha}_{i j} \psi_{j}+\sum_{j=1}^{2} \hat{\beta}_{i j} \omega_{j}+\hat{\gamma}_{i} n_{i}+\hat{\delta}_{i} d, \\
-\operatorname{div}\left(\vec{u}_{0}^{(i)} \xi_{j}^{(i)}\right)+\tau_{i} \xi_{j}^{(i)}=\operatorname{div}\left(\vec{w}^{(i)} \zeta_{j 1}^{(i)}\right)+\tau_{i j} d \text { in } \Omega, \\
\vec{w}^{(i)}=0 \text { on } \Omega, \psi_{i}=0 \text { on } \sum_{\dot{n}}^{i}, \omega_{i}=\Pi \omega_{i}, \xi_{j}^{(i)}=0 \text { on } \Sigma_{\text {out }}^{i}, \\
n_{i}=\sum_{k=1}^{2} \chi_{i k}^{0}\left[\int_{\Omega}^{[}\left[\tilde{\delta}_{k} d+\sum_{j=1}^{2}\left(\tilde{\alpha}_{k j} \psi_{j}+\widetilde{\beta}_{k j} \omega_{j}+\tilde{\gamma}_{k j} \xi_{j}^{(k)}\right)\right] d x, i, j=1,2 .\right.
\end{gathered}
$$

These equations use the following notations

$$
\begin{gathered}
\mathcal{A}_{k}(\vec{w})=\mathcal{A}\left(\vec{w} ; \mathbf{N}_{k}\right), \mathcal{B}_{k}(\rho, \vec{u}, \vec{w})=\mathcal{B}\left(\rho, \vec{u}, \vec{w} ; \mathbf{N}_{k}\right), \mathcal{S}_{k}(\vec{w})=\mathcal{S}\left(\vec{w} ; \mathbf{N}_{k}\right), k=0,1, \\
\mathcal{L}\left(\psi_{i}, \vec{w}^{(i)}\right)=\mathcal{B}_{0}\left(\psi_{i}, \vec{u}_{0}^{(i)}, \vec{u}_{0}^{(i)}\right)+\mathcal{B}_{0}\left(\rho_{i}^{1}, \vec{w}^{(i)}, \vec{u}_{0}^{(i)}\right)+\mathcal{B}_{0}\left(\rho_{i}^{1}, \vec{u}_{1}^{(i)}, \vec{w}^{(i)}\right), \\
\mathcal{E}=\mathcal{S}_{0}\left(\vec{u}_{1}^{(2)}-\vec{u}_{1}^{(1)}\right)-\mathcal{S}_{1}\left(\vec{u}_{1}^{(2)}-\vec{u}_{1}^{(1)}\right), \\
\mathcal{D}_{i}=\sum_{j=1}^{2} \mu_{i j}\left(\mathcal{A}_{0}\left(\vec{u}_{1}^{(j)}\right)-\mathcal{A}_{1}\left(\vec{u}_{1}^{(j)}\right)\right)+\operatorname{Re}\left(\mathcal{B}_{0}\left(\rho_{i}^{1}, \vec{u}_{1}^{(i)}, \vec{u}_{1}^{(i)}\right)-\mathcal{B}_{1}\left(\rho_{i}^{1}, \vec{u}_{1}^{(i)}, \vec{u}_{1}^{(i)}\right)\right), \\
d=g_{0}-g_{1}, g_{k}=\sqrt{\operatorname{det} \mathbf{N}_{k}} .
\end{gathered}
$$

Symbols $\chi_{i k}^{0}$ refer to elements of the matrix $\left(k\left(N_{0}\right) I-A\left(N_{0}, \vec{\theta}_{0}\right)^{-1}\right.$. Coefficients $\alpha_{i j}, \beta_{i j}, \gamma_{i}, \delta_{i}$, $\hat{\alpha}_{i j}, \hat{\beta}_{j}, \hat{\gamma}_{i}, \hat{\delta}_{i}, \quad \tilde{\alpha}_{i j}, \widetilde{\beta}_{i j}, \tilde{\gamma}_{i j}, \widetilde{\delta}_{i}, i, j=1,2$, in the righthand members of equations (9), (10) and (13) depend on solutions $\vec{q}_{0}$ and $\vec{q}_{1}$ and are considered as known functions. Expressions for these coefficients are very bulky, whereby they are not stated here. Let us point out only their estimates that will be required in the future. Under the conditions of Theorem 1, the inequalities

$$
\begin{gathered}
\left\{\left\|\alpha_{i j}\right\|_{X^{s, r}}, i \neq j,\left\|\beta_{j}\right\|_{X^{s, r}},\left\|\delta_{i}\right\|_{X^{s, r},},\left\|\hat{\alpha}_{i j}\right\|_{X^{s, r}},\left\|\hat{\beta}_{i j}\right\|_{X^{s, r}},\left\|\hat{\delta}_{i}\right\|_{X^{s, r}}\right\} \leq c \tau, \\
\left\{\begin{array}{l}
\left.\left\|\widetilde{\alpha}_{i j}\right\|_{X^{s, r}},\left\|\widetilde{\beta}_{j}\right\|_{X^{s, r}},\left\|\tilde{\gamma}_{i j}\right\|_{X^{s, r}}\left\|\widetilde{\delta}_{i}\right\|_{X^{s, r}}\right\} \leq c \tau, \\
\left\|\alpha_{i i}\right\|_{X^{s, r}},\left\|\gamma_{i}\right\|_{X^{s, r}},\left\|\hat{\gamma}_{i}\right\|_{X^{s, r}}
\end{array}\right\} \leq c, i, j=1,2,
\end{gathered}
$$

are valid. Parameter $\tau$ indicates the radius of a ball in the space $V^{s, r} \times X^{s, r}$, to which solutions $\vec{\theta}_{0}$ and $\vec{\theta}_{1}$ belong. $c$ denotes a constant depending only on the domain $\Omega$, vector fields $\vec{U}^{(1)}, \vec{U}^{(2)}$ and parameters $r, s$, $\tau_{11}, \tau_{22}$. Subsequently dependence of a particular constant on $\Omega, \vec{U}^{(1)}, \vec{U}^{(2)}, \tau_{1}, \tau_{2}$ will be referred to as the dependence on the problem data.

2. Conjugate problem. The feature of the problem (8)-(13) for the difference $\vec{q}_{0}-\vec{q}_{1}$ is that the known results [7] on transport equations are not applicable to equations (10), because the summand $\vec{w}^{(i)} \cdot \nabla \varphi_{i}^{1}$ does not satisfy the required smoothness conditions (is neither smooth nor bounded). However, the existence theorem implies that summands $\vec{w}^{(i)} \cdot \nabla \varphi_{i}^{1}$ belong to space $\mathcal{W}^{s-1, r}(\Omega)$, dual to $\mathcal{W}_{0}^{1-s, r^{\prime}}(\Omega)$, and therefore it is possible to treat the difference $\vec{q}_{0}-\vec{q}_{1}$ as a very weak solution to the problem (8)-(13). In this regard, let us formulate the conjugate problem as follows.

For given vector fields $\vec{H}^{(i)}$, scalar fields $G_{i}, F_{i}, M_{1 i}, M_{2 i}$ and constants $s_{i}, i=1,2$, it is necessary to find the vector fields $\vec{w}_{*}^{(i)}$, scalar fields $\omega_{i}^{*}, \psi_{i}^{*}, \xi_{i}^{(j)^{*}}$ and constants $n_{i}^{*}, i, j=1,2$, such that

$$
\left.\sum_{j=1}^{2} \mu_{i j}\left(\Delta \vec{w}_{*}^{(j)}-\nabla \omega_{j}^{*}-\mathcal{A}_{0}\left(\vec{w}_{*}^{(j)}\right)\right)-\operatorname{Re} \mathcal{H}_{i}\left(\vec{w}_{*}^{(i)}\right)+(-) 1^{i+1} \mathcal{S}_{0}\left(\vec{w}_{*}^{(2)}-\vec{w}_{*}^{(1)}\right)+\psi_{i}^{*} \cdot \nabla \varphi_{i}^{1}+\sum_{j=1}^{2} \zeta_{j 1}^{(i)} \cdot \nabla \xi_{j}^{(i)}\right)^{*} \vec{H}^{(i)} \text { in } \Omega
$$




$$
\begin{gathered}
\operatorname{div} \vec{w}_{*}^{(i)}=\Pi \sum_{k=1}^{2}\left[\hat{\beta}_{k i} \psi_{k}^{*}+\sum_{j=1}^{2}\left(n_{k}^{*} \chi_{k j}^{0} \widetilde{\beta}_{j i}+\mu_{k j} \beta_{j i} \omega_{k}^{*}\right)\right]+\Pi G_{i} \text { in } \Omega, \\
-\operatorname{div}\left(\psi_{i}^{*} \vec{u}_{0}^{(i)}\right)+\tau_{i i} \psi_{i}^{*}=\operatorname{Re}_{i}\left(\vec{w}_{*}^{(i)}\right)+\sum_{k=1}^{2}\left[\hat{\alpha}_{k i} \psi_{k}^{*}+\sum_{j=1}^{2}\left(n_{k}^{*} \chi_{k j}^{0} \widetilde{\alpha}_{j i}+\mu_{k j} \alpha_{j i} \omega_{k}^{*}\right)\right]+F_{i} \text { in } \Omega, \\
\vec{u}_{0}^{(i)} \nabla \xi_{j}^{(i)^{*}}+\tau_{i i} \xi_{j}^{(i)^{*}}=\tilde{\gamma}_{i j} \sum_{k=1}^{2} n_{k}^{*} \chi_{k i}^{0}+M_{j i} \text { in } \Omega, \\
n_{i}^{*}=\int_{\Omega}^{(}\left(\gamma_{i} \sum_{j=1}^{2} \mu_{j i} \omega_{j}^{*}+\hat{\gamma}_{i} \psi_{i}^{*}\right) d x+s_{i}, \\
\vec{w}_{*}^{(i)}=0 \text { on } \partial \Omega, \psi_{i}^{*}=0 \text { on } \Sigma_{\text {out }}^{i}, \xi_{j}^{(i)^{*}}=0 \text { on } \Sigma_{\text {in }}^{i}, \Pi \omega_{i}^{*}=\omega_{i}^{*}, i, j=1,2 .
\end{gathered}
$$

Linear operators $\mathcal{H}_{i}$ and $\mathcal{M}_{i}$ are defined by the following formulas

$$
\begin{gathered}
\mathcal{H}_{i}(\vec{h})=\rho_{i}^{1} \nabla\left(\mathbf{N}_{0}^{-1} \vec{u}_{0}^{(i)}\right) \mathbf{N}_{0}^{-1} \vec{h}-\left(\mathbf{N}_{0}^{T}\right)^{-1} \operatorname{div}\left(\rho_{i}^{1} \vec{u}_{1}^{(i)} \otimes\left(N_{0}^{-1} \vec{h}\right)\right), \\
\mathcal{M}_{i}(\vec{h})=\left(\vec{u}_{0}^{(i)} \nabla\left(\mathbf{N}_{0}^{-1} \vec{u}_{0}^{(i)}\right) \cdot \mathbf{N}_{0}^{-1} \vec{h}, i=1,2 .\right.
\end{gathered}
$$

The further content of this section is devoted to proving existence and uniqueness of strong and weak solutions of the conjugate problem (15)-(20). These results enable us to derive estimates of norms for the differences $\vec{w}^{(i)}, \omega_{i}, \psi_{i}, \xi_{i}^{(j)}, n_{i}$.

Theorem 2. Let the conditions of the existence theorem are satisfied and parameters $s, r$ are such that $\frac{1}{2}<s<1,(1-s) r>3$. Then there are numbers $c, \sigma_{c}$ and $\tau_{c}$ depending on the objective and parameters $s, r$ such that if $\min \left\{\tau_{1}, \tau_{2}\right\}>\sigma_{c}$ and $0<\tau \leq \tau_{c}$, then for every vector function $\vec{f}=\left(\vec{f}^{(1)}, \vec{f}^{(2)}\right)$, $\vec{f}^{(i)}=\left(\vec{H}^{(i)} ; G_{i}, F_{i}, M_{1 i}, M_{2 i} ; s_{i}\right) \in \mathcal{U}^{s, r}$, the problem (15)-(20) has a unique solution $\vec{h}=\left(\vec{h}^{(1)}, \vec{h}^{(2)}\right)$,

$$
A\left[\vec{h}_{*}\right]-B\left[\vec{h}_{*}\right]=\vec{F}, \vec{h}_{*}=\left(\vec{h}_{*}^{(1)}, \vec{h}_{*}^{(2)}\right) \vec{h}_{*}^{(i)}=\left(\vec{w}_{*}^{(i)} ; \omega_{i}^{*}, \psi_{i}^{*}, \xi_{1}^{(i)^{*}}, \xi_{2}^{(i)^{*}} ; n_{i}^{*}\right)
$$

where the integral-differential operators $A$ and $B$ are determined by the formulas

$$
\begin{gathered}
A\left[\vec{h}_{*}\right]=\left(A_{1}\left[\vec{h}_{*}\right], A_{2}\left[\vec{h}_{*}\right]\right), \\
A_{i}\left[\vec{h}_{*}\right]=\left\{\begin{array}{l}
\sum_{j=1}^{2} \mu_{j i}\left(\Delta \vec{w}_{*}^{(j)}-\nabla \omega_{j}^{*}\right)+\sum_{j=1}^{2} \zeta_{j 1}^{(i)} \cdot \nabla \xi_{j}^{(i)^{*}} \\
\operatorname{div} \vec{w}_{*}^{(i)} \\
-\vec{u}_{0}^{(i)} \nabla \psi_{i}^{*}+\tau_{i i} \psi_{i}^{*}-\sum_{k=1}^{2} \sum_{j=1}^{2} \mu_{k j} \alpha_{j i} \omega_{k}^{*} \\
\vec{u}_{0}^{(i)} \nabla \xi_{1}^{(i)^{*}}+\tau_{i i} \xi_{1}^{(i)^{*}} \\
\vec{u}_{0}^{(i)} \nabla \xi_{2}^{(i)}+\tau_{i i} \xi_{2}^{(i)^{*}} \\
\left.n_{i}^{*}-\int_{\Omega}^{\int} \int_{j}^{2} \gamma_{j=1} \mu_{j i}^{*} \omega_{j}^{*}+\hat{\gamma}_{i} \psi_{i}^{*}\right) d x \\
B\left[\vec{h}_{*}\right]=\left(B_{1}\left[\vec{h}_{*}\right], B_{2}\left[\vec{h}_{*}\right]\right),
\end{array}\right\}
\end{gathered}
$$




$$
B_{i}\left[\vec{h}_{*}\right]=\left\{\begin{array}{l}
\sum_{j=1}^{2} \mu_{j i} \mathcal{A}_{0}\left(\vec{w}_{*}^{(j)}\right)+\operatorname{Re} \mathcal{H}_{i}\left(\vec{w}_{*}^{(i)}\right)+(-1)^{i} \mathcal{S}_{0}\left(\vec{w}_{*}^{(2)}-\vec{w}_{*}^{(1)}\right)-\psi_{i}^{*} \cdot \nabla \varphi_{i}^{1} \\
\Pi \sum_{k=1}^{2}\left[\hat{\beta}_{k i} \psi_{k}^{*}+\sum_{j=1}^{2}\left(n_{k}^{*} \chi_{k j}^{0} \tilde{\beta}_{j i}+\mu_{k j} \beta_{j i} \omega_{k}^{*}\right)\right] \\
\operatorname{Re} \mathcal{M}_{i}\left(\vec{w}_{*}^{(i)}\right)+\psi_{i}^{*} \operatorname{div} \vec{u}_{0}^{(i)}+\sum_{k=1}^{2}\left[\hat{\alpha}_{k i} \psi_{k}^{*}+n_{k}^{*} \chi_{k j}^{0} \tilde{\alpha}_{j i}\right] \\
\tilde{\gamma}_{i 1} \sum_{k=1}^{2} n_{k}^{*} \chi_{k i}^{0} \\
\tilde{\gamma}_{i 2} \sum_{k=1}^{2} n_{k}^{*} \chi_{k i}^{0} \\
0
\end{array}\right\}
$$

Let us consider the following boundary value problem by connecting to the system of equations

$$
A\left[\vec{h}_{*}\right]=\vec{F}
$$

the boundary conditions

$$
\vec{w}_{*}^{(j)}=0 \text { on } \partial \Omega, \psi_{i}^{*}=0 \text { on } \Sigma_{\text {out }}^{i}, \xi_{1}^{(i)^{*}}=0, \xi_{2}^{(i)^{*}}=0 \text { on } \Sigma_{\text {in }}^{i}, \quad \Pi \omega_{i}^{*}=\omega_{i}^{*}, i=1,2 .
$$

It is easy to see that for each right-hand member $\vec{F}=\left(\vec{F}^{(1)}, \vec{F}^{(2)}\right), \quad \vec{F}^{(i)}=\left(\vec{H}^{(i)} ; G_{i}, F_{i}, M_{1 i}, M_{2 i} ; s_{i}\right)$ of the space $\mathcal{U}^{s, r}$, the boundary value problem (24), (25) is divided into several independent linear boundary value problems, namely, functions $\xi_{j}^{(i)^{*}}, i, j=1,2$, are defined independently as solutions to the following problem for transport equation

$$
\begin{aligned}
& \vec{u}_{0}^{(i)} \nabla \xi_{j}^{(i)^{*}}+\tau_{i i} \xi_{j}^{(i)^{*}}=M_{j i} \text { in } \Omega, \xi_{j}^{(i)^{*}}=0 \text { on } \sum_{i n}^{i}, i, j=1,2 . \\
& \qquad-\vec{u}_{0}^{(i)} \nabla \psi_{i}^{*}+\tau_{i i} \psi_{i}^{*}=\sum_{k=1}^{2} \sum_{j=1}^{2} \mu_{k j} \alpha \\
& \text { The procedure for building solution to the problem (24), } \\
& \text { (25) ends with finding the constants } n_{i}^{*} \text { according to the } \\
& \text { formulas } \\
& \qquad n_{i}^{*}=\iint_{\Omega}\left(\gamma_{i} \sum_{j=1}^{2} \mu_{j i} \omega_{j}^{*}+\hat{\gamma}_{i} \psi_{i}^{*}\right) d x+s_{i}, i=1,2 .
\end{aligned}
$$$$
-\vec{u}_{0}^{(i)} \nabla \psi_{i}^{*}+\tau_{i i} \psi_{i}^{*}=\sum_{k=1}^{2} \sum_{j=1}^{2} \mu_{k j} \alpha_{j i} \omega_{k}^{*}+F_{i} \text { in } \Omega, \psi_{i}^{*}=0 \text { on } \Sigma_{\text {out }}^{i}, i=1,2 \text {. }
$$

On the basis of known results on transport equations and linear Stokes-type problem $[7,26,9]$ we can assert the existence of the constants $c$ and $\sigma_{c}$, depending on the problem data $\Omega, \vec{U}^{(1)}, \vec{U}^{(2)}$ and parameters $r, s$, such that if $\min \left\{\tau_{1}, \tau_{2}\right\} \geq \sigma_{c}$, then the problem (24), (25) is uniquely solvable in spaces $\mathcal{V}^{s, r}$ and $\mathcal{F}^{s, r}$ and the following inequality is valid

$$
\left\|\overrightarrow{h_{*}}\right\|_{\mathcal{V}^{s, r}} \leq c\|\vec{F}\|_{\mathcal{U}^{s, r}},\left\|\overrightarrow{h_{*}}\right\|_{\mathcal{F}^{s, r}} \leq c\|\vec{F}\|_{\mathcal{E}^{s, r}}
$$

The foregoing can be interpreted in the form of existence of (inverse) bounded linear operator $A^{-1}: \mathcal{U}^{s, r} \rightarrow \mathcal{V}^{s, r}$ $\left(A^{-1}: \mathcal{E}^{s, r} \rightarrow \mathcal{F}^{s, r}\right)$ which assigns a unique solution $\vec{h}_{*}=A^{-1}[\vec{F}]$ to the boundary value problem (24), (25) to the element $\vec{F} \in \mathcal{U}^{s, r}\left(\vec{F} \in \mathcal{E}^{s, r}\right)$.

Let us now turn to the integral-differential operator $B$ given by the formulas (23), and note the following properties.

Lemma 1. Let us assume that conditions of Theorem 1 and $\frac{1}{2}<s<1,(1-s) r>3$ are satisfied. Then $B$ is a bounded linear operator from $\mathcal{V}^{s, r}$ to $\mathcal{U}^{s, r}$ and from $\mathcal{F}^{s, r}$ to $\mathcal{E}^{s, r}$, and the following inequalities are valid
After this, the components $\vec{w}_{*}^{(i)}, \omega_{i}^{*}, i=1,2$, are found as solutions to the Stokes-type problems, i.e.

$$
\begin{gathered}
\sum_{j=1}^{2} \mu_{j i}\left(\Delta \vec{w}_{*}^{(j)}-\nabla \omega_{j}^{*}\right)=\vec{H}^{(i)}-\sum_{j=1}^{2} \zeta_{j 1}^{(i)} \cdot \nabla \xi_{j}^{(i)^{*}} \text { in } \Omega, \\
\operatorname{div} \vec{w}_{*}^{(i)}=\Pi G_{i} \text { in } \Omega, \\
\vec{w}_{*}^{(i)}=0 \text { on } \partial \Omega, \Pi \omega_{i}^{*}=\omega_{i}^{*}, i=1,2 .
\end{gathered}
$$

The next step is definition of components $\psi_{i}^{*}$ as the solution to the boundary value problem

$$
\begin{aligned}
& \|B \vec{h}\|_{\mathcal{U}^{s, r}} \leq c \cdot \tau\|\vec{h}\|_{\mathcal{V}^{s, r}}, \\
& \|B \vec{h}\|_{\mathcal{E}^{s, r}} \leq c \cdot \tau\|\vec{h}\|_{\mathcal{F}^{s, r}} .
\end{aligned}
$$

Here, the constant $c$ depends only on $\Omega$, vector fields $\vec{U}^{(1)}, \vec{U}^{(2)}$ and parameters $r, s$.

Now we can complete the proof of existence and uniqueness of the conjugate problem solution.

Let us assume that conditions of Theorem 2 are satisfied. The conjugate problem (15)-(20) can be written as the operator equation

$$
\left(I-A^{-1} B\right) \vec{h}=A^{-1} \vec{f}
$$

Due to (26), (27) linear operators $A^{-1}: \mathcal{U}^{s, r} \rightarrow \mathcal{V}^{s, r}$ and $A^{-1} B: \mathcal{V}^{s, r} \rightarrow \mathcal{V}^{s, r}$ are bounded, moreover

$$
\left\|A^{-1} B\right\|_{L\left(\mathcal{V}^{s, r}\right)} \leq c_{V} \cdot \tau,
$$

where the constant $c_{V}$ depends only on the problem data and parameters $r, s$. On the basis of the conditions in Theorem 1, parameter $\tau$ satisfies the conditions $0<\tau \leq \tau^{*}\left(\tau_{1}, \tau_{2}\right)$. Let us choose the constant $\tau_{c}$ such that the inequalities are valid

$$
0<\tau_{c} \leq \tau^{*}\left(\tau_{1}, \tau_{2}\right) \tau_{c} \cdot c_{V} \leq q<1
$$

From (29) it follows that the norm $\left\|A^{-1} B\right\|_{L\left(\mathcal{V}^{s, r}\right)}$ for $\tau \in\left(0 ; \tau_{c}\right]$ is separated from the unity, and on the basis of the well-known theorem, the operator $\left(I-A^{-1} B\right)^{-1}$ exists and is bounded

$$
\left\|\left(I-A^{-1} B\right)^{-1}\right\|_{L\left(\mathcal{V}^{s, r}\right)} \leq \frac{1}{1-q} .
$$


So for these values $\tau$ and any $\vec{f} \in \mathcal{U}^{s, r}$ the conjugate problem (15)-(20) has a unique solution $\vec{h} \in \mathcal{V}^{s, r}$ and the inequality $(21)$ is valid.

Suppose now that the right-hand member $\vec{f}$ of the problem (15)-(20) belongs to space $\mathcal{E}^{s, r}$. As the operator $A^{-1}$ of the solution to problem (24), (25) is a bounded linear operator from $\mathcal{E}^{s, r}$ to $\mathcal{F}^{s, r}$, then due to estimates (26), (28), we have

$$
\left\|A^{-1} B\right\|_{L\left(\mathcal{F}^{s, r}\right)} \leq c_{F} \cdot \tau .
$$

If the parameter $\tau$ is subordinate to the condition $\tau \in\left(0 ; \tau_{c}\right]$, where

$$
\begin{aligned}
0<\tau_{c} \leq \tau^{*} & \left(\tau_{11}, \tau_{22}\right), \tau_{c} \cdot c_{F} \leq q<1, \\
& \sum_{i=1}^{2}\left[\left\langle\vec{H}^{(i)}, \vec{w}^{(i)}\right\rangle_{1}+\left\langle\omega_{i}, G_{i}\right\rangle_{0}+\left\langle\psi_{i}, F_{i}\right\rangle_{0}+\left\langle\xi_{1}^{(i)}, M_{1 i}\right\rangle_{0}+\left\langle\xi_{2}^{(i)}, M_{2 i}\right\rangle_{0}+n_{i} s_{i}\right]= \\
& =\sum_{i=1}^{2} \int_{\Omega}\left[\vec{w}_{*}^{(i)}\left(\mathcal{D}_{i}+(-1)^{i} \mathcal{E}\right)+d\left(\hat{\delta}_{i} \psi_{i}^{*}+\sum_{j=1}^{2}\left(\tau_{j i} \xi_{j}^{(i)^{*}}+n_{i}^{*} \chi_{i j}^{0} \tilde{\delta}_{j}+\mu_{i j} \delta_{j} \omega_{i}^{*}\right)\right] d x\right.
\end{aligned}
$$

where $\vec{f}^{(i)}=\left(\vec{H}^{(i)} ; G_{i}, F_{i}, M_{1 i}, M_{2 i} ; s_{i}\right), i=1,2$, are arbitrary functions of $\mathcal{W}^{s-1} r(\Omega) \times W^{s, r}(\Omega) \times \mathbb{R}$, and $\left(\vec{w}_{*}^{(i)} ; \omega_{i}^{*}, \psi_{i}^{*}, \xi_{1}^{(i)^{*}}, \xi_{2}^{(i)^{*}} ; n_{i}^{*}\right), i=1,2$, is the relevant solution to the conjugate problem (15)-(20).

Proof. Since the set $L^{r}(\Omega) \times W^{1, r}(\Omega) \times \mathbb{R}$ is contained in $\mathcal{E}^{s, r}$ and is dense in $\mathcal{U}^{s, r}=\mathcal{W}^{s-1, r}(\Omega) \times W^{s, r}(\Omega) \times \mathbb{R}$, then for any element $\vec{f}=\left(\vec{f}^{(1)}, \vec{f}^{(2)}\right), \quad \vec{f}^{(i)}=\left(\vec{H}^{(i)} ; G_{i}, F_{i}, M_{1 i}, M_{2 i} ; s_{i}\right)$ of space $\mathcal{U}^{s, r}$, there is a sequence $\vec{f}_{n}=\left(\vec{f}_{n}^{(1)}, \vec{f}_{n}^{(2)}\right)$, $\vec{f}_{n}^{(i)}=\left(\vec{H}_{n}^{(i)} ; G_{\dot{n}}, F_{\dot{n}}, M_{1 \dot{n}}, M_{2 \dot{n}} ; s_{\dot{\boldsymbol{n}}}\right)$, $\vec{f}_{n}^{(i)} \in L^{r}(\Omega) \times W^{1, r}(\Omega) \times \mathbb{R}$ such that

$$
\vec{f}_{n} \rightarrow \vec{f} \dot{n} \mathcal{U}^{s, r}, n \rightarrow \infty .
$$

Therefore let us begin with prove of the identity (30) for a vector function $\vec{f}$ from a space $L^{r}(\Omega) \times W^{1 r}(\Omega) \times \mathbb{R}$. In this case, the relevant solution $\left(\vec{w}_{*}^{(i)}, \omega_{i}^{*}, \psi_{i}^{*}, \xi_{1}^{(i)^{*}}, \xi_{2}^{(i)^{*}}, n_{i}^{*}\right), i=1,2, \quad$ to the conjugate problem (15)-(20) belongs to the class then, similar to the previous case we obtain that under condition $\vec{f} \in \mathcal{E}^{s, r}$ solution $\vec{h}$ to the problem (15)-(20) belongs to the class $\mathcal{F}^{s, r}$ and the estimate (22) is valid. Theorem 2 is proved.

3. Estimates of differences. For $s \in(0,1), r \in(1, \infty)$ let $\langle\cdot, \cdot\rangle_{1}$ and $\langle\cdot, \cdot\rangle_{0}$ denote the duality relationship between pairs of spaces $\mathcal{W}^{s-1} r(\Omega), \mathcal{W}_{0}^{1-s, r^{\prime}}(\Omega)$ and $\mathbb{W}^{-s, r^{\prime}}(\Omega), W^{s, r}(\Omega)$, respectively.

Lemma 2. Let us assume that conditions of Theorem 2 are satisfied. Then vector fields $\vec{w}^{(j)}, j=1,2$, scalar fields $\omega_{j}, \psi_{j}, \xi_{j}^{(i)}, i, j=1,2$, and constants $n_{j}, j=1,2$, defined according to the formulas (7) belong to spaces $\mathcal{W}_{0}^{1-s, r^{\prime}}(\Omega), \mathbb{W}^{-s, r^{\prime}}(\Omega), \mathbb{R}$ and satisfy the identity
$V^{s, r} \times X^{s, r} \times \mathbb{R}=\mathcal{F}^{s, r}$ (in particular, this means that vector fields $\vec{w}_{*}^{(i)}$ have continuous first-order derivatives and square summable second-order derivatives, and scalar functions $\omega_{i}^{*}, \psi_{i}^{*}, \xi_{1}^{(i)^{*}}, \xi_{2}^{(i)^{*}}$ are continuous and have square summable first-order derivatives). Due to Theorem 1, we can assert that $\vec{w}^{(i)}, \vec{v}_{k}^{(i)}, \vec{u}_{k}^{(i)} \in V^{s, r}$, $\omega_{i}, \psi_{i}, \xi_{j}^{(i)}, \rho_{i}^{k}, \pi_{i}^{k}, \varphi_{i}^{k}, \zeta_{j k}^{(i)} \in X^{s, r}, i, j=1,2, k=0,1$.

The continuity of embeddings $V^{s, r}$ in $C^{1}(\Omega)$ provides continuous differentiability in $\Omega$ vector fields $\vec{w}^{(i)}, \vec{v}_{k}^{(i)}, \vec{u}_{k}^{(i)}$ and, of course, their membership of $W^{2,2}(\Omega)$. Continuity of embedding $X^{s, r}$ in $C(\Omega)$ implies continuity of scalar functions $\omega_{i}, \psi_{i}, \xi_{j}^{(i)}$, $\rho_{i}^{k}, \pi_{i}^{k}, \varphi_{i}^{k}, \zeta_{j k}^{(i)}$, belonging to the same spaces $W^{1,2}(\Omega)$. This implies that equations (8)-(11), (13) are satisfied in the strong sense. Multiplying these equations by the solution $\vec{w}_{*}^{(i)}, \omega_{i}^{*}, \psi_{i}^{*}, \xi_{1}^{(i)^{*}}, \xi_{2}^{(i)^{*}}, n_{i}^{*}, i=1,2$, to the conjugate problem (15)-(20), corresponding to the element $\vec{f} \in L^{r}(\Omega) \times W^{1 r}(\Omega) \times \mathbb{R}$, and integrating by parts, we obtain the identity

$$
\begin{gathered}
\left.\sum_{i=1}^{2}\left[\int \vec{w}_{\Omega}^{(i)} \vec{H}^{(i)}+\omega_{i} G_{i}+\psi_{i} F_{i}+\xi_{1}^{(i)} M_{1 i}+\xi_{2}^{(i)} M_{2 i}\right) d x+n_{i} s_{i}\right]= \\
=\sum_{i=1}^{2} \int_{\Omega}\left[\vec{w}_{*}^{(i)}\left(\mathcal{D}_{i}+(-1)^{i} \mathcal{E}\right)+d\left(\hat{\delta}_{i} \psi_{i}^{*}+\sum_{j=1}^{2}\left(\tau_{j i} \xi_{j}^{(i)^{*}}+n_{i}^{*} \chi_{i j}^{0} \tilde{\delta}_{j}+\mu_{i j} \delta_{j} \omega_{i}^{*}\right)\right] d x .\right.
\end{gathered}
$$

Let us note that vector functions $\left(\vec{H}^{(i)} ; \omega_{i}, \psi_{i}, \xi_{1}^{(i)}, \xi_{2}^{(i)}\right)$ and $\left(\vec{w}^{(i)} ; G_{i}, F_{i}, M_{1 i}, M_{2 i}\right)$ belong to dual spaces $\mathcal{W}^{s-1, r}(\Omega) \times \mathbb{W}^{-s, r^{\prime}}(\Omega) \quad$ and $\quad \mathcal{W}_{0}^{1-s, r^{\prime}}(\Omega) \times W^{s, r}(\Omega)$, respectively. $\quad$ Indeed, $\quad \vec{H}^{(i)} \in \mathcal{W}^{s-1, r}(\Omega)$ because $\vec{H}^{(i)} \in L^{r}(\Omega)$, and there is a limited embedding $L^{r}(\Omega)$ in $\mathcal{W}^{s-1, r}(\Omega)$. Scalar fields $\omega_{i}, \psi_{i}, \xi_{1}^{(i)}, \xi_{2}^{(i)}$ are elements $W^{s, r}(\Omega)$, and embeddings $W^{s, r}(\Omega) \subset L^{r}(\Omega) \subset \mathbb{W}^{-s, r^{\prime}}(\Omega)$ $\left(r>3,1<r^{\prime}=\frac{r}{r-1}<r\right)$ imply that these functions belong to $\mathbb{W}^{-s, r^{\prime}}(\Omega)$.

Further, the vector field $\vec{w}^{(i)} \in V^{s, r}$ and hence $\vec{w}^{(i)} \in C^{1}(\Omega)$. In addition $\vec{w}^{(i)}$ vanishes in $\partial \Omega$. Thus, it is clear that $\vec{w}^{(i)} \in W_{0}^{1, r^{\prime}}(\Omega)$. Since $\mathcal{W}_{0}^{1-s, r^{\prime}}(\Omega)$ is the interpolation space $\left[L^{r^{\prime}}(\Omega) W_{0}^{1, r^{\prime}}(\Omega)\right]_{1-s, r^{\prime}}$ and $W_{0}^{1, r^{\prime}}(\Omega) \subset L^{r^{\prime}}(\Omega)$, then $W_{0}^{1, r^{\prime}}(\Omega) \subset \mathcal{W}_{0}^{1-s, r^{\prime}}(\Omega)$. So, 
we have $\vec{w}^{(i)} \in \mathcal{W}_{0}^{1-s, r^{\prime}}(\Omega)$. On the other hand elements $G_{i}, F_{i}, M_{1 i}, M_{2 i}$ belong to $W^{s, r}(\Omega)$. In view of the above, integrals in the left-hand member of the equality (32) can be written in terms of duality forms and thus, the identity (30) of Lemma 2 is proved for vector functions $\left(\vec{H}^{(i)} ; G_{i}, F_{i}, M_{1 i}, M_{2 i} ; s_{i}\right)$ from a narrower functional space $L^{r}(\Omega) \times W^{1} r(\Omega) \times \mathbb{R}$, i.e, we may write

$$
\begin{aligned}
& \sum_{i=1}^{2}\left\{\left\langle\vec{H}_{n}^{(i)}, \vec{w}^{(i)}\right\rangle_{1}+\left\langle\omega_{i}, G_{i n}\right\rangle_{0}+\left\langle\psi_{i}, F_{i n}\right\rangle_{0}+\left\langle\xi_{1}^{(i)}, M_{1 i n}\right\rangle_{0}+\left\langle\xi_{2}^{(i)}, M_{2 i n}\right\rangle_{0}+n_{i} s_{i n}\right\}= \\
& =\sum_{i=1}^{2}\left\{\vec{w}_{\Omega}^{(i)}\left(\mathcal{D}_{i}+(-1)^{i} \mathcal{E}\right)+d\left(\hat{\delta_{i} \psi_{i n}^{*}}+\sum_{j=1}^{2}\left(\tau_{j i} \xi_{j n}^{(i)^{*}}+n_{i n}^{*} \chi_{i j}^{0} \widetilde{\delta}_{j}+\mu_{i j} \delta_{j} \omega_{i n}^{*}\right)\right)\right\} d x,
\end{aligned}
$$

where $\vec{h}_{n}^{(i)}=\left(\vec{w}_{n^{*}}^{(i)} ; \omega_{\dot{n}}^{*}, \psi_{\dot{n}}^{*}, \xi_{1 n}^{(i)^{*}}, \xi_{2 n}^{(i)^{*}} ; n_{\dot{n}}^{*}\right), i=1,2$, is the solution to the problem (15)-(20), corresponding to the "right-hand member" $\vec{f}_{n}^{(i)}, i=1,2$. From (31), due to Theorem 2, we have that

$$
\vec{h}_{n}=\left(\vec{h}_{n}^{(1)}, \vec{h}_{n}^{(2)}\right) \rightarrow \vec{h}=\left(\vec{h}^{(1)}, \vec{h}^{(2)}\right) \dot{\boldsymbol{n}} \mathcal{V}^{s, r}, n \rightarrow \infty
$$

Passing to the limit as $n \rightarrow \infty$ in (33), due to (31),
(34) we obtain the identity(30) stated in Lemma 2. Lemma 2 is proved.

Identity (30) proved in Lemma 2 means that the difference $\vec{q}_{0}-\vec{q}_{1}$ is a very weak solution to the linear problem (8)-(13).

Lemma 3. Let the conditions of Lemma 2 are satisfied. Then the following inequality is valid

$$
\begin{gathered}
\sum_{i=1}^{2}\left(\left\|\vec{w}^{(i)}\right\|_{\mathcal{W}_{0}^{1-s, r^{\prime}}}+\left\|\psi_{i}\right\|_{\mathbb{W}^{-s, r^{\prime}}}+\left\|\omega_{i}\right\|_{\mathbb{W}^{-s, r^{\prime}}}+\left\|\xi_{1}^{(i)}\right\|_{\mathbb{W}^{-s, r^{\prime}}}+\left\|\xi_{2}^{(i)}\right\|_{\mathbb{W}^{-s, r^{\prime}}}+\left|n_{i}\right|\right) \leq \\
\leq c\left(\|d\|_{L^{1}(\Omega)}+\left\|\mathcal{D}_{1}\right\|_{L^{1}(\Omega)}+\left\|\mathcal{D}_{2}\right\|_{L^{1}(\Omega)}+\|\mathcal{E}\|_{L^{1}(\Omega)}\right)
\end{gathered}
$$

where the constant $c$ depends on the problem data and parameters $r, s$.

Proof. Turning to the equality (30), we note that since the coefficients $\hat{\delta}_{i}, \widetilde{\delta}_{i}, \delta_{i}$ are bounded

$$
\sum_{i=1}^{2}\left(\left\|\vec{w}_{*}^{(i)}\right\|_{C(\Omega)}+\left\|\psi_{i}^{*}\right\|_{C(\Omega)}+\left\|\omega_{i}^{*}\right\|_{C(\Omega)}+\left\|\xi_{1}^{(i)^{*}}\right\|_{C(\Omega)}+\left\|\xi_{2}^{(i)^{*}}\right\|_{C(\Omega)}+\left|n_{i}^{*}\right|\right) \cdot\left(\|d\|_{L^{1}(\Omega)}+\left\|\mathcal{D}_{1}\right\|_{L^{1}(\Omega)}+\left\|\mathcal{D}_{2}\right\|_{L^{1}(\Omega)}+\|\mathcal{E}\|_{L^{1}(\Omega)}\right) \cdot c
$$

In addition, due to embedding theorem and estimate (21) we have

$$
\sum_{i=1}^{2}\left(\left\|\vec{w}_{*}^{(i)}\right\|_{C(\Omega)}+\left\|\psi_{i}^{*}\right\|_{C(\Omega)}+\left\|\omega_{i}^{*}\right\|_{C(\Omega)}+\left\|\xi_{1}^{(i)^{*}}\right\|_{C(\Omega)}+\left\|\xi_{2}^{(i)^{*}}\right\|_{C(\Omega)}+\left|n_{i}^{*}\right|\right) \leq c\|\vec{h}\|_{\mathcal{V}^{s, r}} \leq c\|\vec{f}\|_{\mathcal{U}^{s, r}}
$$

From (36) and (37) we obtain

$$
\left|\sum_{i=1}^{2}\left\{\left\langle\vec{H}^{(i)}, \vec{w}^{(i)}\right\rangle_{1}+\left\langle\omega_{i}, G_{i}\right\rangle_{0}+\left\langle\psi_{i}, F_{i}\right\rangle_{0}+\left\langle\xi_{1}^{(i)}, M_{1 i}\right\rangle_{0}+\left\langle\xi_{2}^{(i)}, M_{2 i}\right\rangle_{0}+n_{i} s_{i}\right\}\right| \leq c \sum_{i=1}^{2}\left(\|d\|_{L^{1}(\Omega)}+\left\|\mathcal{D}_{i}\right\|_{L^{1}(\Omega)}+\|\mathcal{E}\|_{L^{1}(\Omega)}\right)\|\vec{f}\|_{\mathcal{U}^{s, r}}
$$

The inequality (38) obviously implies the estimate (35). Lemma 3 is proved.

The inequality (35) implies, firstly, the uniqueness of solution to the problem (6). In addition, (35) implies that the mapping associating the matrix-valued function $N$ with solution $\vec{q}$ to the inhomogeneous boundary value problem (6) is a Lipschitz solution in a weak norm.

\section{MATERIAL DERIVATIVES}

So-called material derivatives of solution to the problem (6) is an important tool to prove the drag functional differentiability. This section is devoted to their study. Solution $\vec{q}=\left(\vec{v}^{(1)}, \vec{v}^{(2)} ; \pi_{1}, \pi_{2}, \varphi_{1}, \varphi_{2}, \zeta_{1}^{(1)}, \zeta_{2}^{(1)}, \zeta_{1}^{(2)}, \zeta_{2}^{(2)} ; m_{1}, m_{2}\right)$ to the problem (6) is a function of spatial variable $x=\left(x_{1}, x_{2}, x_{3}\right) \in \Omega$ and parameter $\varepsilon$. Let us assume that $\vec{q}(\varepsilon)=\langle\langle x \rightarrow q(x, \varepsilon)\rangle\rangle$ and consider $\vec{q}$ (and, of course, components $\vec{q}$ ) as a function of $\varepsilon$ with values in the space of functions of $x$.

Material derivative $\quad \vec{q}^{\prime}(0)=\left(\vec{w}^{(1)}, \vec{w}^{(2)} ; \omega_{1}, \omega_{2}, \psi_{1}, \psi_{2}, \xi_{1}^{(1)}, \xi_{2}^{(1)}, \xi_{1}^{(2)}, \xi_{2}^{(2)} ; n_{1}, n_{2}\right) \quad$ of $\quad$, solution $\vec{q}(\varepsilon)=\left(\vec{v}^{(1)}(\varepsilon), \vec{v}^{(2)}(\varepsilon) ; \pi_{1}(\varepsilon), \pi_{2}(\varepsilon), \varphi_{1}(\varepsilon), \varphi_{2}(\varepsilon), \zeta_{1}^{(1)}(\varepsilon), \zeta_{2}^{(1)}(\varepsilon), \zeta_{1}^{(2)}(\varepsilon), \zeta_{2}^{(2)}(\varepsilon) ; m_{1}(\varepsilon), m_{2}(\varepsilon)\right)$ to the problem (6) with $\varepsilon=0$ is defined as follows:

$$
\begin{gathered}
\vec{w}^{(j)}=\lim _{\varepsilon \rightarrow 0} \vec{w}^{(j)} \varepsilon, \omega_{j}=\lim _{\varepsilon \rightarrow 0} \omega_{j \varepsilon}, \psi_{j}=\lim _{\varepsilon \rightarrow 0} \psi_{j \varepsilon}, \xi_{j}^{(i)}=\lim _{\varepsilon \rightarrow 0} \xi_{j \varepsilon}^{(i)}, n_{j}=\lim _{\varepsilon \rightarrow 0} n_{j \varepsilon} \quad i, j=1,2 . \\
\vec{w}_{\varepsilon}^{(j)}=-\varepsilon^{-1}\left(\vec{v}^{(j)}(0)-\vec{v}^{(j)}(\varepsilon)\right), \omega_{j \varepsilon}=-\varepsilon^{-1}\left(\pi_{j}(0)-\pi_{j}(\varepsilon)\right), \psi_{j \varepsilon}=-\varepsilon^{-1}\left(\varphi_{j}(0)-\varphi_{j}(\varepsilon)\right), \\
\xi_{j \varepsilon}^{(i)}=-\varepsilon^{-1}\left(\zeta_{j}^{(i)}(0)-\zeta_{j}^{(i)}(\varepsilon)\right), n_{j \varepsilon}=-\varepsilon^{-1}\left(m_{j}(0)-m_{j}(\varepsilon)\right), i, j=1,2,
\end{gathered}
$$

provided that the limits in (39) exist in some sense. 
Equations for material derivatives can be easily obtained by formal use of $\vec{\theta}=\vec{\theta}(\varepsilon)$ and $\mathbf{N}=\mathbf{N}(\varepsilon)$ in equations (6) and differentiating results according to $\varepsilon$ at zero. This formal procedure gives the following system of equations and boundary conditions for material derivatives.

$$
\begin{gathered}
\sum_{j=1}^{2} \mu_{i j} \Delta \vec{w}^{(j)}-\nabla \omega_{i}=\mathfrak{L}^{*}\left(\psi_{i}, \vec{w}^{(i)}\right)+\mathcal{D}_{i}^{*}+(-1)^{i}\left(\mathcal{E}^{*}+a\left(\vec{w}^{(2)}-\vec{w}^{(1)}\right)\right) \text { in } \Omega, \\
\operatorname{div} \vec{w}^{(i)}=\sum_{j=1}^{2} \alpha_{i j}^{*} \psi_{j}+\sum_{j=1}^{2} \beta_{i j}^{*} \omega_{j}+\gamma_{i}^{*} n_{i}+\delta_{i}^{*} d^{*} \text { in } \Omega, \\
\vec{u}^{(i)}(0) \cdot \nabla \psi_{i}+\tau_{i i} \psi_{i}=-\vec{w}^{(i)} \cdot \nabla \varphi_{i}(0)+\sum_{j=1}^{2} \hat{\alpha}_{i j}^{*} \psi_{j}+\sum_{j=1}^{2} \hat{\beta}_{i j}^{*} \omega_{j}+\hat{\gamma}_{i}^{*} n_{i}+\hat{\delta}_{i}^{*} d^{*} \text { in } \Omega, \\
-\operatorname{div}\left(\vec{u}^{(i)}(0) \xi_{j}^{(i)}\right)+\tau_{i i} \xi_{j}^{(i)}=\operatorname{div}\left(\vec{w}^{(i)} \zeta_{j}^{(i)}(0)\right)+\tau_{j i} d^{*} \text { in } \Omega, \\
\vec{w}^{(i)}=0 \text { on } \partial \Omega, \quad \psi_{i}=0 \text { on } \Sigma_{i n}^{i}, \quad \omega_{i}=\Pi \omega_{i}, \quad \xi_{j}^{(i)}=0 \text { on } \Sigma_{o u t}^{i}, \\
n_{i}=\sum_{k=1}^{2} \chi_{i k}^{0}\left[\int_{\Omega}^{[}\left[\widetilde{\delta}_{k}^{*} d^{*}+\sum_{j=1}^{2}\left(\widetilde{\alpha}_{k j}^{*} \psi_{j}+\widetilde{\beta}_{k j}^{*} \omega_{j}+\widetilde{\gamma}_{k j}^{*} \xi_{j}^{(k)}\right)\right] d x, i, j=12 .\right.
\end{gathered}
$$

These equations use the following notations

$$
\begin{gathered}
\mathcal{D}_{i}^{*}=-\operatorname{Re} \rho_{i}(0)\left(\vec{u}^{(i)}(0) \cdot \nabla\left(\mathbb{D} \vec{u}^{(i)}(0)\right)+\mathbb{D}^{\mathbb{T}} \vec{u}^{(i)}(0) \cdot \nabla \vec{u}^{(i)}(0)\right)+\sum_{j=1}^{2} \mu_{i j}\left(\operatorname{div}\left(\mathbb{T} \nabla \vec{u}^{(i)}(0)\right)+\mathbb{D}^{\mathbb{T}} \Delta \vec{u}^{(i)}(0)+\Delta\left(\mathbb{D} \vec{u}^{(i)}(0)\right)\right), \\
\mathfrak{L}^{*}\left(\psi_{i}, \vec{w}^{(i)}\right)=\operatorname{Re}\left(\psi_{i} \vec{u}^{(i)}(0) \cdot \nabla \vec{u}^{(i)}(0)+\rho_{i}(0) \vec{w}^{(i)} \cdot \nabla \vec{u}^{(i)}(0)+\rho_{i} \vec{u}^{(i)}(0) \cdot \nabla \vec{w}^{(i)}\right) ; \\
\mathcal{E}^{*}=a \mathbb{T}\left(\vec{u}^{(2)}(0)-\vec{u}^{(1)}(0)\right) ; \quad d^{*}=\operatorname{div} \vec{T} ; \quad \mathbb{D}=\operatorname{div} \vec{T} \mathbf{I}-D \vec{T} ; \quad \mathbb{T}=\operatorname{div} \vec{T} \mathbf{I}-D \vec{T}-(D \vec{T})^{T} .
\end{gathered}
$$

Coefficients $\alpha_{i j}^{*}, \beta_{i j}^{*}, \gamma_{i}^{*}, \delta_{i}^{*}, \hat{\alpha}_{i j}^{*}, \hat{\beta}_{i j}^{*}, \hat{\gamma}_{i}^{*}, \hat{\delta}_{i}^{*}, \widetilde{\alpha}_{i j}^{*}, \widetilde{\beta}_{i j}^{*}, \tilde{\gamma}_{i j}^{*}, \widetilde{\delta}_{i}^{*}, i, j=1,2$, in the right-hand members of equations (40)depend on solution to the problem (6), corresponding to $\varepsilon=0$.

To prove existence of a very weak solution of the problem (40)-(41) let us introduce the conjugate problem in the following formulation: for given vector fields $\vec{H}^{(i)}$, scalar fields $G_{i}, F_{i}, M_{1 i}, M_{2 i}$ and constants $s_{i}, i=12$, it is necessary to find vector fields $\vec{w}_{*}^{(i)}$, scalar fields $\omega_{i}^{*}, \psi_{i}^{*}, \xi_{i}^{(j)^{*}}$ constants $n_{i}^{*}, i, j=12$, such that

$$
\begin{gathered}
\sum_{j=1}^{2} \mu_{j i}\left(\Delta \vec{w}_{*}^{(j)}-\nabla \omega_{j}^{*}\right)-\operatorname{Re} \mathrm{H}_{i}^{*}\left(\vec{w}_{*}^{(j)}\right)+(-1)^{i+1} a\left(\vec{w}_{*}^{(2)}-\vec{w}_{*}^{(1)}\right)+\psi_{i}^{*} \cdot \nabla \varphi_{i}(0)+\sum_{j=1}^{2} \zeta_{j}^{(i)}(0) \nabla \xi_{j}^{(i)^{*}}=\vec{H}^{(i)} \text { in } \Omega, \\
\operatorname{div} \vec{w}_{*}^{(i)}=\Pi \sum_{k=1}^{2}\left[\hat{\beta}_{k i}^{*} \psi_{k}^{*}+\sum_{j=1}^{2}\left(n_{k}^{*} \chi_{k j}^{0} \widetilde{\beta}_{j i}^{*}+\mu_{k j} \beta_{j i}^{*} \omega_{k}^{*}\right)\right]+\Pi G_{i} \text { in } \Omega, \\
-\operatorname{div}\left(\psi_{i}^{*} \vec{u}^{(i)}(0)\right)+\tau_{i i} \psi_{i}^{*}=\operatorname{Re} \mathrm{M}_{i}^{*}\left(\vec{w}_{*}^{(j)}\right)+\sum_{k=1}^{2}\left[\hat{\alpha}_{k i}^{*} \psi_{k}^{*}+\sum_{j=1}^{2}\left(n_{k}^{*} \chi_{k j}^{0} \widetilde{\alpha}_{j i}^{*}+\mu_{k j} \alpha_{j i}^{*} \omega_{k}^{*}\right)\right]+F_{i} \text { in } \Omega, \\
\vec{u}^{(i)}(0) \nabla \xi_{j}^{(i)^{*}}+\tau_{i i} \xi_{j}^{(i)^{*}}=\widetilde{\gamma}_{i j}^{*} \sum_{k=1}^{2} n_{k}^{*} \chi_{k i}^{0}+M_{j i} \text { in } \Omega, \\
n_{i}^{*}=\int_{\Omega}\left(\gamma_{i}^{*} \sum_{j=1}^{2} \mu_{j i} \omega_{j}^{*}+\hat{\gamma}_{i}^{*} \psi_{i}^{*}\right) d x+s_{i}, i, j=1,2, \\
\vec{w}_{*}^{(j)}=0 \text { on } \partial \Omega, \quad \psi_{i}^{*}=0 \text { on } \Sigma_{\text {out }}^{i}, \quad \xi_{j}^{(i) *}=0 \text { on } \Sigma_{i n}^{i}, \Pi \omega_{i}^{*}=\omega_{i}^{*}, i, j=1,2 .
\end{gathered}
$$

Linear operators $\mathcal{H}_{i}^{*}$ and $\mathcal{M}_{i}^{*}$ are defined by the following formulas

$$
\mathcal{H}_{i}^{*}(\vec{h})=\rho_{i}(0) \nabla\left(\vec{u}^{(i)}(0)\right) \vec{h}-\operatorname{div}\left(\rho_{i}(0) \vec{u}^{(i)}(0) \otimes \vec{h}\right), \mathcal{M}_{i}^{*}(\vec{h})=\left(\vec{u}^{(i)}(0) \nabla \vec{u}^{(i)}(0)\right) \cdot \vec{h}, i=12 .
$$

Well-posedness of the conjugate problem (42) statement is the result of the following lemma.

Lemma 4. Let us assume that conditions of Theorem 2 are satisfied. Then for each vector function $\vec{f}=\left(\vec{f}^{(1)}, \vec{f}^{(2)}\right), \quad \vec{f}^{(i)}=\left(\vec{H}^{(i)} ; G_{i}, F_{i}, M_{1 i}, M_{2 i} ; s_{i}\right) \in \mathcal{U}^{s, r}$, the problem (42) has a unique solution $\vec{h}_{*}=\left(\vec{h}_{*}^{(1)}, \vec{h}_{*}^{(2)}\right)$, $\vec{h}_{*}^{(i)}=\left(\vec{w}_{*}^{(i)} ; \omega_{i}^{*}, \psi_{i}^{*}, \xi_{1}^{(i)^{*}}, \xi_{2}^{(i)^{*}} ; n_{i}^{*}\right) \in \mathcal{V}^{s, r}$.

Proof. Problem (42) is a special case of the problem(15)-(20), with $N_{0}=\mathrm{I}$ and $\mathrm{g}_{0}=1, \vec{q}_{i}=\vec{q}(0) \vec{u}_{i}^{(j)}=\vec{u}_{*}^{(j)}+\vec{v}^{(j)}(0)$, 
$\rho_{j}^{i}=\rho_{j}^{*}+\varphi_{j}(0), \quad \zeta_{j i}^{(k)}=\zeta_{j}^{(k)}(0) \quad i=0,1 ; k, j=1,2$.

Therefore, the lemma is a direct consequence of Theorem 2.

Linear equations (40) are obtained by formal differentiation, and the fact that the limits $\vec{w}^{(i)}, \omega_{i}, \psi_{i}, \xi_{j}^{(i)}, n_{i}, i, j=1,2$ in (39) really exist and satisfy (40), is not obvious. Further it will be shown that they exist in a weak sense, are defined as the elements of the space $\mathcal{W}_{0}^{1-s, r^{\prime}}(\Omega) \times \mathbb{W}^{-s, r^{\prime}}(\Omega) \times \mathbb{R}$ and defined as a very weak solution of the linear problem (40).

The proof of these facts is preceded by a brief comment of a technical nature.

Lemma 5. There are numbers $\varepsilon_{1}>0$ and $c, c_{1}$, depending on $\|\vec{T}\|_{C^{2}(\Omega)}$ such that for any $\varepsilon \in\left[0 ; \varepsilon_{1}\right]$, the following representations exist

$$
\mathbf{N}(\varepsilon)^{ \pm 1}=\mathbf{I} \pm \varepsilon \mathbb{D}+\varepsilon^{2} \mathbb{D}^{ \pm}, \mathfrak{g}(\varepsilon)^{ \pm 1}=1 \pm \varepsilon \operatorname{div} \vec{T}+\varepsilon^{2} \mathfrak{g}^{ \pm}
$$

where $\mathbb{D}=\operatorname{div} \vec{T} \mathbf{I}-D \vec{T}$ and $\left\|\mathbb{D}^{ \pm}\right\|_{C^{2}(\Omega)^{+}}\left\|\mathfrak{g}^{ \pm}\right\| \leq c$, and deviations $\mathbf{N}(\varepsilon)^{ \pm 1}-\mathbf{I}, \mathfrak{g}(\varepsilon)^{ \pm 1}-1$ satisfy the inequality

$$
\left\|\mathbf{N}(\varepsilon)^{ \pm 1}-\mathbf{I}\right\|_{C^{2}(\Omega)}+\left\|\mathfrak{g}(\varepsilon)^{ \pm 1}-1\right\|_{C^{2}(\Omega)} \leq c_{1} \varepsilon
$$

The proof of Lemma 5 is, for example, in [7].

Theorem 3. Let the conditions of Theorem 2 and $\varepsilon \in\left[0 ; \varepsilon_{*}\right]$ are satisfied, where $\varepsilon_{*}=\min \left\{c_{1}^{-1} \tau^{2}, \varepsilon_{1}\right\}$ and the constants $c_{1}$ and $\varepsilon_{1}$ are chosen in accordance with Lemma 5. Then there are such $\vec{h}=\left(\vec{h}^{(1)}, \vec{h}^{(2)}\right)$ $\vec{h}^{(i)}=\left(\vec{w}^{(i)} ; \omega_{i}, \psi_{i}, \xi_{i}^{(1)}, \xi_{i}^{(2)} ; n_{i}\right) \in \mathcal{W}_{0}^{1-s, r^{\prime}}(\Omega) \times \mathbb{W}^{-s, r^{\prime}}(\Omega) \times \mathbb{R}$ that $\quad \vec{w}_{\varepsilon}^{(i)} \rightarrow \vec{w}^{(i)} \quad$ weakly $\quad$ в $\quad \mathcal{W}_{0}^{1-s, r^{\prime}}(\Omega), i=1,2$, $\left(\omega_{i \varepsilon}, \psi_{i_{\varepsilon}}, \xi_{i \varepsilon}^{(1)}, \xi_{i \varepsilon}^{(2)}\right) \rightarrow\left(\omega_{i}, \psi_{i}, \xi_{i}^{(1)}, \xi_{i}^{(2)}\right) \quad$ weakly in $\mathbb{W}^{-s, r^{\prime}}(\Omega), i=1,2, \quad n_{i \varepsilon} \rightarrow n_{i} \quad \hat{a} \quad \mathbb{R}$ at $\varepsilon \rightarrow 0$. In addition, forany $\left(\vec{H}^{(i)} ; G_{i}, F_{i}, M_{1 i}, M_{2 i} ; e_{i}\right) \in \mathcal{U}^{s, r}, i=1,2$, the identity is valid

$$
\begin{aligned}
& \sum_{i=1}^{2}\left\{\left\langle\vec{H}^{(i)}, \vec{w}^{(i)}\right\rangle_{1}+\left\langle\omega_{i}, G_{i}\right\rangle_{0}+\left\langle\psi_{i}, F_{i}\right\rangle_{0}+\left\langle\xi_{1}^{(i)}, M_{1 i}\right\rangle_{0}+\left\langle\xi_{2}^{(i)}, M_{2 i}\right\rangle_{0}+n_{i} \mathrm{~s}_{i}\right\}= \\
& =\sum_{i=1}^{2}\left\{\vec{w}_{\Omega} \vec{w}_{*}^{(i)}\left(\mathcal{D}_{i}^{*}+(-1)^{i} \mathcal{E}^{*}\right)+d^{*}\left(\hat{\delta}_{i}^{*} \psi_{i}^{*}+\sum_{j=1}^{2}\left(\tau_{j i} \xi_{j}^{(i)}+n_{i}^{*} \chi_{i j}^{0} \widetilde{\delta}_{j}^{*}+\mu_{i j} \delta_{j}^{*} \omega_{i}^{*}\right)\right)\right\} d x,
\end{aligned}
$$

Here $\vec{h}_{*}=\left(\vec{h}_{*}^{(1)}, \vec{h}_{*}^{(2)}\right), \vec{h}_{*}^{(i)}=\left(\vec{w}_{*}^{(i)} ; \omega_{i}^{*}, \psi_{i}^{*}, \xi_{1}^{(i) *}, \xi_{2}^{(i)^{*}} ; n_{i}^{*}\right) \in \mathcal{V}^{s, r}$ is the solution to problem (42) defined in Lemma 4; $\mathcal{D}_{i}^{*}$, $\mathcal{E}^{*}$ and $d^{*}$ are defined by the folrmulas (41).

We confine ourselves to a short scheme of proof of Theorem 3. Establishing the boundedness of sequence of functions $\vec{h}_{\varepsilon}=\left(\vec{h}_{\varepsilon}^{(1)}, \vec{h}_{\varepsilon}^{(2)}\right)$, $\vec{h}_{\varepsilon}^{(i)}=\left(\vec{w}_{\varepsilon}^{(i)} ; \omega_{i \varepsilon}, \psi_{i_{\varepsilon}}, \xi_{i \varepsilon}^{(1)}, \xi_{i \varepsilon}^{(2)} ; n_{i \varepsilon}\right)$ in reflexive space $\mathcal{W}_{0}^{1-s, r^{\prime}}(\Omega) \times \mathbb{W}^{-s, r^{\prime}}(\Omega) \times \mathbb{R}$ and passing if necessary to a subsequence, we obtain existence of vector functions $\left(\vec{w}^{(i)} ; \omega_{i}, \psi_{i}, \xi_{i}^{(1)}, \xi_{i}^{(2)} ; n_{i}\right) \in \mathcal{W}_{0}^{1-s, r^{\prime}}(\Omega) \times \mathbb{W}^{-s, r^{\prime}}(\Omega) \times \mathbb{R}$

$$
\begin{aligned}
& \sum_{i=1}^{2}\left\{\left\langle\vec{H}^{(i)}, \vec{w}_{\varepsilon}^{(i)}\right\rangle_{1}+\left\langle\omega_{i \varepsilon}, G_{i}\right\rangle_{0}+\left\langle\psi_{i \varepsilon}, F_{i}\right\rangle_{0}+\left\langle\xi_{1 \varepsilon}^{(i)}, M_{1 i}\right\rangle_{0}+\left\langle\xi_{2 \varepsilon}^{(i)}, M_{2 i}\right\rangle_{0}+n_{i \varepsilon} s_{i}\right\}= \\
& =-\frac{1}{\varepsilon} \sum_{i=1}^{2}\left\{\int_{\Omega} \vec{w}_{*_{\varepsilon}}^{(i)} \cdot\left(\mathcal{D}_{i}(\varepsilon)+(-1)^{i} \mathcal{E}(\varepsilon)\right)+d(\varepsilon)\left(\hat{\delta}_{i}(\varepsilon) \psi_{i \varepsilon}^{*}+\sum_{j=1}^{2}\left(\tau_{j i} \xi_{j n}^{(i)^{*}}+n_{i \varepsilon}^{*} \chi_{i j} \tilde{\delta}_{j}(\varepsilon)+\mu_{i j} \delta_{j}(\varepsilon) \omega_{i \varepsilon}^{*}\right)\right) d x\right\} \text {, }
\end{aligned}
$$

where $\quad \vec{h}_{* \varepsilon}^{(i)}=\left(\vec{w}_{* \varepsilon}^{(i)} ; \omega_{i \varepsilon}^{*}, \psi_{i \varepsilon}^{*}, \xi_{1 n}^{(i)^{*}}, \xi_{2 \varepsilon}^{(i)^{*}} ; n_{i \varepsilon}^{*}\right), i=1,2$ is the solution to problem the conjugate to the problem for the function $\vec{h}_{\varepsilon}=\left(\vec{h}_{\varepsilon}^{(1)}, \vec{h}_{\varepsilon}^{(2)}\right)$, $\vec{h}_{\varepsilon}^{(i)}=\left(\vec{w}_{\varepsilon}^{(i)} ; \omega_{i \varepsilon}, \psi_{i_{\varepsilon}}, \xi_{i \varepsilon}^{(1)}, \xi_{i \varepsilon}^{(2)} ; n_{i \varepsilon}\right)$. Since space

$$
\begin{aligned}
& \sum_{i=1}^{2}\left\{\left\langle\vec{H}^{(i)}, \vec{w}_{\varepsilon}^{(i)}\right\rangle_{1}+\left\langle\omega_{i \varepsilon}, G_{i}\right\rangle_{0}+\left\langle\psi_{i \varepsilon}, F_{i}\right\rangle_{0}+\left\langle\xi_{1 \varepsilon}^{(i)}, M_{1 i}\right\rangle_{0}+\left\langle\xi_{2 \varepsilon}^{(i)}, M_{2 i}\right\rangle_{0}+n_{i \varepsilon} s_{i}\right\} \rightarrow \\
& \rightarrow \sum_{i=1}^{2}\left\{\left\langle\vec{H}^{(i)}, \vec{w}^{(i)}\right\rangle_{1}+\left\langle\omega_{i}, G\right\rangle_{0}+\left\langle\psi_{i}, F_{i}\right\rangle_{0}+\left\langle\xi_{1}^{(i)}, M_{1 i}\right\rangle_{0}+\left\langle\xi_{2}^{(i)}, M_{2 i}\right\rangle_{0}+n_{i} s_{i}\right\} \text { as } \varepsilon \rightarrow 0 .
\end{aligned}
$$

Further, by setting a weak convergence of sequence of solutions $\vec{h}_{*_{\varepsilon}}=\left(\vec{h}_{*_{\varepsilon}}^{(1)}, \vec{h}_{*_{\varepsilon}}^{(2)}\right)$, $\vec{h}_{*_{\varepsilon}}^{(i)}=\left(\vec{w}_{*_{\varepsilon}}^{(i)} ; \omega_{i \varepsilon}^{*}, \psi_{i \varepsilon}^{*}, \xi_{1 n}^{(i)^{*}}, \xi_{2 \varepsilon}^{(i)^{*}} ; n_{i \varepsilon}^{*}\right)$, to the conjugate problem in space $W^{s+1, r}(\Omega) \times W^{s, r}(\Omega) \times \mathbb{R}$ to the solution $\vec{h}_{*}=\left(\vec{h}_{*}^{(1)}, \vec{h}_{*}^{(2)}\right), \quad \vec{h}_{*}^{(i)}=\left(\vec{w}_{*}^{(i)} ; \omega_{i}^{*}, \psi_{i}^{*}, \xi_{1}^{(i)^{*}}, \xi_{2}^{(i)^{*}} ; n_{i}^{*}\right)$, of the problem (42) and considering the compactness of 
embedding $W^{s+1} r(\Omega) \times W^{s, r}(\Omega) \times \mathbb{R}$ in $C(\Omega) \times \mathbb{R}$ we obtain that $\vec{h}_{*_{\varepsilon}} \rightarrow \vec{h}_{*} \dot{i} C(\Omega) \times \mathbb{R}, i=1,2$.

Properties of coefficients of equations for difference

relations $\left(\vec{w}_{\varepsilon}^{(i)} ; \omega_{i \varepsilon}, \psi_{i \varepsilon}, \xi_{i \varepsilon}^{(1)}, \xi_{i \varepsilon}^{(2)} ; n_{i \varepsilon}\right) i=1,2$, allow to pass to the limit in the right-hand member of the identity(46) and to state that

$$
\begin{gathered}
-\frac{1}{\varepsilon} \sum_{i=1}^{2}\left\{\int_{\Omega} \vec{w}_{\psi_{\varepsilon}}^{(i)} \cdot\left(\mathcal{D}_{i}(\varepsilon)+(-1)^{i} \mathcal{E}(\varepsilon)\right)+d(\varepsilon)\left(\hat{\delta}_{i}(\varepsilon) \psi_{i \varepsilon}^{*}+\sum_{j=1}^{2}\left(\tau_{j i} \xi_{j \varepsilon}^{(i)}+n_{i \varepsilon}^{*} \chi_{i j} \widetilde{\delta}_{j}(\varepsilon)+\mu_{i j} \delta_{j}(\varepsilon) \omega_{i \varepsilon}^{*}\right)\right) d x\right\} \rightarrow \\
\rightarrow \sum_{i=1}^{2} \int_{\Omega}\left\{\vec{w}_{*}^{(i)}\left(\mathcal{D}_{i}^{*}+(-1)^{i} \mathcal{E}^{*}\right)+d^{*}\left(\hat{\delta}_{i}^{*} \psi_{i}^{*}+\sum_{j=1}^{2}\left(\tau_{j i} \xi_{j}^{(i)^{*}}+n_{i}^{*} \chi_{i j}^{0} \widetilde{\delta}_{j}^{*}+\mu_{i j} \delta_{j}^{*} \omega_{i}^{*}\right)\right)\right\} d x
\end{gathered}
$$

Equations (46)-(48) prove the identity(45).

Thus it is proved that every weak limit $\vec{h}=\left(\vec{h}^{(1)}, \vec{h}^{(2)}\right), \vec{h}^{(i)}=\left(\vec{w}^{(i)} ; \omega_{i}, \psi_{i}, \xi_{i}^{(1)}, \xi_{i}^{(2)} ; n_{i}\right)$, of the sequence $\vec{h}_{\varepsilon}=\left(\vec{h}_{\varepsilon}^{(1)}, \vec{h}_{\varepsilon}^{(2)}\right), \quad \vec{h}_{\varepsilon}^{(i)}=\left(\vec{w}_{\varepsilon}^{(i)} ; \omega_{i_{\varepsilon}}, \psi_{i_{\varepsilon}}, \xi_{i \varepsilon}^{(1)}, \xi_{i \varepsilon}^{(2)} ; n_{i \varepsilon}\right)$ satisfies the identity (45). It is necessary to demonstrate that the sequence has a unique weak limit point, and hence converges weakly to this point. Let us assume that there are $\left(\vec{w}^{(i)} ; \omega_{i}, \psi_{i}, \xi_{i}^{(1)}, \xi_{i}^{(2)} ; n_{i}\right), i=1,2$, and $\left(\vec{w}^{\prime(i)} ; \omega_{i}^{\prime}, \psi_{i}^{\prime}, \xi_{i}^{(1)}, \xi_{i}^{\prime(2)} ; n_{i}^{\prime}\right), i=1,2$, of $\mathcal{W}_{0}^{1-s, r^{\prime}}(\Omega) \times \mathbb{W}^{-s, r^{\prime}}(\Omega) \times \mathbb{R}$ that satisfy (45). Then the following identity is valid for all $\left(\vec{H}^{(i)}, G_{i}, F_{i}, M_{1 i}, M_{2 i}, s_{i}\right) \in \mathcal{W}^{s-1, r}(\Omega) \times W^{s, r}(\Omega) \times \mathbb{R}, i=1,2$,

$$
\sum_{i=1}^{2}\left\{\left\langle\vec{H}^{(i)}, \vec{w}^{(i)}-\vec{w}^{(i)}\right\rangle_{1}+\left\langle\omega_{i}-\omega_{i}^{\prime}, G_{i}\right\rangle_{0}+\left\langle\psi_{i}-\psi_{i}^{\prime}, F_{i}\right\rangle_{0}+\left\langle\xi_{1}^{(i)}-\xi_{1}^{\prime(i)}, M_{1 i}\right\rangle_{0}+\left\langle\xi_{2}^{(i)}-\xi_{2}^{(i)}, M_{2 i}\right\rangle_{0}+\left(n_{i}-n_{i}^{\prime}\right) s_{i}\right\}=0
$$

Since the space $\mathcal{W}_{0}^{s-1, r}(\Omega) \times W^{s, r}(\Omega) \times \mathbb{R}$ is dual to $\mathcal{W}_{0}^{1-s, r^{\prime}}(\Omega) \times \mathbb{W}^{-s, r^{\prime}}(\Omega) \times \mathbb{R}$, this identity implies that $\left(\vec{w}^{(i)} ; \omega_{i}, \psi_{l}, \xi_{i}^{(1)}, \xi_{l}^{(2)} ; n_{i}\right)=\left(\vec{w}^{\prime(i)} ; \omega_{l}^{\prime}, \psi_{l}^{\prime}, \xi_{l}^{\prime(1)}, \xi_{l}^{(2)} ; n_{l}^{\prime}\right), i=1,2$.

The following lemma if the consequence of the results obtained in the proof of Theorem 3

Lemma 6. Let us assume that conditions of Theorem 2 are satisfied. Then $\vec{q}(\varepsilon) \rightarrow \vec{q}(0)$ in $W^{s+1, r}(\Omega) \times W^{s, r}(\Omega) \times \mathbb{R}$ as $\varepsilon \rightarrow 0$.

\section{DRAG FUNCTIONAL DOMAIN DERIVATIVE}

Let us assume the conditions of Theorem 3 are satisfied. Then each matrix $\mathbf{N}(\varepsilon)$ corresponds to a unique solution $\left.\vec{\theta}(\varepsilon)=\left(\vec{v}^{()}()_{\varepsilon}\right),{ }^{(}(\varepsilon) ; \pi_{1}(\varepsilon), \pi_{2}(\varepsilon), \varphi_{1}(\varepsilon), \varphi_{2}(\varepsilon)\right)$ to the problem (6), which in turn induces the solution

$$
\vec{u}^{(i)}(\varepsilon)=\vec{u}_{*}^{(i)}+\vec{v}^{(i)}(\varepsilon), \rho_{i}(\varepsilon)=\rho_{i}^{*}+\varphi_{i}(\varepsilon), q_{i}(\varepsilon)=q_{i}^{*}+\pi_{i}(\varepsilon)+\Lambda \cdot p_{i}\left(\rho_{i}^{*}\right)+\sum_{j=1} \beta_{i j} m_{j}, i=1,2,
$$

to the problem (5). Turning to the unperturbed domain $\Omega$ in the formula (4) for drag functional, we obtain the following representation.

$$
\begin{aligned}
J_{D} S_{\varepsilon}=J\left(\Omega_{\varepsilon}\right)= & -\sum_{i=1}^{2} U_{\infty} \cdot\left\langle\int_{\Omega} \operatorname{Re\eta } \rho_{i} \vec{u}^{(i)} \nabla\left(\mathbf{N}^{-1} \vec{u}^{(i)}\right) d x+\int_{\Omega}\left(\sum _ { j = 1 } ^ { 2 } \mu _ { i j } g ^ { - 1 } \left(\mathbf{N}^{T} \nabla\left(\mathbf{N}^{-1} \vec{u}^{(j)}\right)+\right.\right.\right. \\
& \left.\left.\left.+\nabla\left(\mathbf{N}^{-1} \vec{u}^{(j)}\right)^{T} \mathbf{N}-\operatorname{div}\left(\vec{u}^{(j)}\right) \mathbb{I}\right)-q_{i} \mathbb{I}\right) \mathbf{N}^{T} \nabla \eta d x\right\rangle
\end{aligned}
$$

where $\eta$ is the arbitrary smooth function identically equal to 1 in the neighborhood of the obstacle $S$ and identically equal to zero in the neighborhood of the boundary $\Sigma$. Substituting functions (49) into the expression (50), we obtain:

$$
J\left(\Omega_{\varepsilon}\right)=\sum_{i=1}^{2}\left(U_{\infty} \cdot J_{1}^{i}\left(\Omega_{\varepsilon}\right)+U_{\infty} \cdot J_{2}^{i}\left(\Omega_{\varepsilon}\right)\right)
$$

where

$$
J_{1}^{i}\left(\Omega_{\varepsilon}\right)=-\int_{\Omega} \operatorname{Re\eta } \rho_{i}(\varepsilon) \vec{u}^{(i)}(\varepsilon) \nabla\left(\mathrm{N}(\varepsilon)^{-1} \vec{u}^{(i)}(\varepsilon)\right) d x
$$

$J_{2}^{\mathrm{i}}\left(\Omega_{\varepsilon}\right)=\int_{\Omega} q_{i}(\varepsilon) \mathbf{N}(\varepsilon)^{T} \nabla \eta d x-\int_{\Omega}\left(\sum_{j=1}^{2} \mu_{i j} g(\varepsilon)^{-1}\left(\mathbf{N}(\varepsilon)^{T} \nabla\left(\mathbf{N}(\varepsilon)^{-1} \vec{u}^{(j)}(\varepsilon)\right)+\nabla\left(\mathbf{N}(\varepsilon)^{-1} \vec{u}^{(j)}(\varepsilon)\right)^{T} \mathbf{N}(\varepsilon)-\operatorname{div}\left(\vec{u}^{(j)}(\varepsilon)\right) \mathbb{I}\right)\right) \mathbf{N}(\varepsilon)^{T} \nabla \eta d x$

Calculation of the drag and its variations with respect to perturbations of $S$ is a problem of practical importance, for example, to build a numerical algorithm for finding the optimal shape of the obstacle in the flow. In this section, an explicit formula for the following derivative is obtained

$$
d(\Omega)[\vec{T}]=\lim _{\varepsilon \rightarrow 0} \frac{1}{\varepsilon}\left(J\left(\Omega_{\varepsilon}\right)-J(\Omega)\right)
$$

Let us calculate $d J(\Omega)[\vec{T}]$ in terms of material derivatives of solutions to the problem (6). The corresponding result is given in the following theorem, 
which is a direct consequence of the Theorem 3 .

Theorem 4. Suppose that all the conditions of Theorem 3 are satisfied. Then derivative $d J(\Omega)[\vec{T}]$ defined by the formula (52) exists and can be represented as

$$
\begin{aligned}
& d(\Omega)[\vec{T}]=L_{u}(\vec{T})+L_{e}(\vec{T}) \\
& B_{i}=\left(\mu_{1 i}+\mu_{2 i}\right) \Delta \eta U_{\infty}+\operatorname{Re\rho }_{i}(0)\left(\vec{u}^{(i)}(0) \cdot \nabla \eta\right) U_{\infty}-\operatorname{Re\rho }_{i}(0) \nabla \vec{u}^{(i)}(0) \cdot U_{\infty} \eta, \\
& A=\nabla \eta \cdot U_{\infty}, \quad C_{i}=-\operatorname{Re\eta }\left(\vec{u}^{(i)}(0) \cdot \nabla \vec{u}^{(i)}(0)\right) \cdot U_{\infty},
\end{aligned}
$$

and $L_{e}$ takes the form

$$
\begin{gathered}
\left.L_{e}(\vec{T})=-\sum_{i=1}^{2} U_{\infty} R e \int_{\Omega} \rho_{i}(0)\left(\vec{u}^{(i)}(0) \cdot \nabla \eta\right) \mathbb{D} \vec{u}^{(i)}(0)\right] d x+ \\
+\sum_{i=1}^{2} U_{\infty} \iint_{\Omega}\left(\sum_{j=1}^{2} \mu_{i j}\left(\nabla\left(\vec{u}^{(j)}(0)\right)+\nabla\left(\vec{u}^{(j)}(0)\right)^{T}-\operatorname{div}\left(\vec{u}^{(j)}(0)\right) \mathbf{I}\right)\right)(D \vec{T})^{T} \nabla \eta d x- \\
\left.-\sum_{i=1}^{2} U_{\infty} \int_{\Omega}\left(\sum_{j=1}^{2} \mu_{i j}\left(\mathbb{D}^{\mathbb{T}} \nabla\left(\vec{u}^{(j)}(0)\right)-\nabla\left(\mathbb{D} \vec{u}^{(j)}(0)\right)+\left(\mathbb{D}^{\mathbb{T}} \nabla\left(\vec{u}^{(j)}(0)\right)-\nabla\left(\mathbb{D} \vec{u}^{(j)}(0)\right)\right)\right)^{T}\right)-q_{i}(0) \mathbb{D}^{\mathbb{T}}\right) \nabla \eta d x,
\end{gathered}
$$

where $\mathbb{D}=\operatorname{div} \vec{T} \mathbf{I}-D \vec{T}$.

Proof of Theorem 4. Substituting relationships

in (51), we obtain the difference relationship

$$
\vec{u}^{(i)}(\varepsilon)-\vec{u}^{(i)}(0)=\varepsilon \vec{w}_{\varepsilon}^{(i)}, \rho_{i}(\varepsilon)-\rho_{i}(0)=\varepsilon \psi_{i_{\varepsilon}}, q_{i}(\varepsilon)-q_{i}(0)=\varepsilon \omega_{i_{\varepsilon}}+\varepsilon \sum_{j=1}^{2} \beta_{i j} n_{\varepsilon j}, i=1,2,
$$

$$
\frac{1}{\varepsilon}\left(J\left(\Omega_{\varepsilon}\right)-J(\Omega)\right)=\sum_{i=1}^{2}\left(U_{\infty} \cdot \frac{1}{\varepsilon}\left(J_{1}^{i}\left(\Omega_{\varepsilon}\right)-J_{1}^{i}(\Omega)\right)+U_{\infty} \cdot \frac{1}{\varepsilon}\left(J_{2}{ }^{i}\left(\Omega_{\varepsilon}\right)-J_{2}{ }^{i}(\Omega)\right)\right),
$$

which can be represented as:

$$
\frac{1}{\varepsilon}\left(J\left(\Omega_{\varepsilon}\right)-J(\Omega)\right)=L_{\varepsilon, u}+L_{\varepsilon, e}
$$

where

$$
\begin{gathered}
L_{\varepsilon, u}=-\sum_{i=1}^{2} \operatorname{Re} U_{\infty} \int_{\Omega} \eta\left[\rho_{i}(\varepsilon) \vec{w}_{\varepsilon}^{(i)} \nabla \vec{u}^{(i)}(\varepsilon)+\psi_{i \varepsilon} \vec{u}^{(i)}(0) \nabla \vec{u}^{(i)}(\varepsilon)+\rho_{i}(0) \vec{u}^{(i)}(0) \nabla \vec{w}_{\varepsilon}^{(i)}\right] d x \\
-\sum_{i=1}^{2} U_{\infty} \iint_{\Omega}\left(\sum_{j=1}^{2} \mu_{i j}\left(\nabla \vec{w}_{\varepsilon}^{(j)}+\nabla\left(\vec{w}_{\varepsilon}^{(j)}\right)^{T}-\operatorname{div}\left(\vec{w}_{\varepsilon}^{(j)}\right) \mathbf{I}\right)\right) \nabla \eta d x+\sum_{i=1}^{2} U_{\infty} \int_{\Omega}\left(\omega_{i_{\varepsilon}}+\sum_{j=1}^{2} \beta_{i j} n_{\varepsilon j}\right) \mathbf{I} \nabla \eta d x \\
\varepsilon L_{\varepsilon, e}=\sum_{i=1}^{2} U_{\infty} \int_{\Omega}\left\{\operatorname{Re\eta }\left[\rho_{i}(\varepsilon) \vec{u}^{(i)}(\varepsilon) \nabla\left(\left(\mathbf{I}-\mathbf{N}(\varepsilon)^{-1}\right) \vec{u}^{(i)}(\varepsilon)\right)\right]+\left(\sum_{j=1}^{2} \mu_{i j}\left(\nabla\left(\vec{u}^{(j)}(\varepsilon)\right)+\nabla\left(\vec{u}^{(j)}(\varepsilon)\right)^{T}-\operatorname{div}\left(\vec{u}^{(j)}(\varepsilon)\right) \mathbf{I}\right)-q_{i}(\varepsilon) \mathbf{I}\right) \nabla \eta\right\} d x \\
-\sum_{i=1}^{2} U_{\infty} \int_{\Omega}\left(\sum_{j=1}^{2} \mu_{i j} g(\varepsilon)^{-1}\left(\mathbf{N}(\varepsilon)^{T} \nabla\left(\mathbf{N}(\varepsilon)^{-1} \vec{u}^{(j)}(\varepsilon)\right)+\nabla\left(\mathbf{N}(\varepsilon)^{-1} \vec{u}^{(j)}(\varepsilon)\right)^{T} \mathbf{N}(\varepsilon)-\operatorname{div}\left(\vec{u}^{(j)}(\varepsilon)\right) \mathbf{I}\right)-q_{i}(\varepsilon) \mathbf{I}\right) \mathbf{N}(\varepsilon)^{T} \nabla \eta d x(59)
\end{gathered}
$$

Note that $\vec{u}^{(i)}(\varepsilon), \vec{u}^{(i)}(0), \vec{w}_{\varepsilon}^{(i)}, i=1,2$ are continuously differentiable in $\Omega$ and belong to the class $W^{2,2}(\Omega)$. In turn, $\rho_{i}(\varepsilon), \rho_{i}(0), \psi_{i_{\varepsilon}}, q_{i}(\varepsilon), q_{i}(0), \omega_{i_{\varepsilon}}, i=1,2 \quad$ are continuous in the domain $\Omega$ and belong to the class
$W^{1,2}(\Omega)$. Thus, taking into account that $\vec{w}_{\varepsilon}^{(i)}, i=1,2$ vanishon $\partial \Omega$ andequations $\operatorname{div}\left(\rho_{i}(0) \cdot \vec{u}^{(i)}(0)\right)=0, i=1,2$ in $\Omega$ are satisfied, we make transformation of the following integrals.

$$
\begin{gathered}
\int_{\Omega} \operatorname{Re} \rho_{i}(0) \vec{u}^{(i)}(0) \cdot \nabla \vec{w}_{\varepsilon}^{(i)} \eta d x=-\int_{\Omega} \operatorname{Re} \rho_{i}(0)\left(\vec{u}^{(i)}(0) \cdot \nabla \eta\right) \vec{w}_{\varepsilon}^{(i)} d x \\
\int_{\Omega}^{2}\left\{\mu_{j=1}\left(\nabla \vec{w}_{\varepsilon}^{(j)}+\nabla\left(\vec{w}_{\varepsilon}^{(j)}\right)^{T}-\operatorname{div}\left(\vec{w}_{\varepsilon}^{(j)}\right) \mathbf{I}\right)\right\} \nabla \eta d x=-\sum_{j=1}^{2} \mu_{i j} \int_{\Omega} \vec{w}_{\varepsilon}^{(j)} \Delta \eta d x .
\end{gathered}
$$

Since the function $\eta$ vanishes in the neighborhood of

$\Sigma$ and is equal to unity in the neighborhood of $\partial S$, then 


$$
\int_{\Omega} \sum_{k=1}^{2} \beta_{i k} n_{k \varepsilon} \nabla \eta d x=-\sum_{k=1}^{2} \beta_{i k} n_{k \varepsilon} \int_{\partial S} \vec{n} d s=0 .
$$

Taking into account the formulas (60) and (61) of the functional $L_{\varepsilon, u}$ from (58), let us equate

$$
\begin{gathered}
B_{i \varepsilon}=\left(\mu_{1 i}+\mu_{2 i}\right) \Delta \eta U_{\infty}+\operatorname{Re} \rho_{i}(0)\left(\vec{u}^{(i)}(0) \cdot \nabla \eta\right) U_{\infty}-\operatorname{Re} \rho_{i}(\varepsilon) \nabla \vec{u}^{(i)}(\varepsilon) \cdot U_{\infty} \eta, \\
A=\nabla \eta \cdot U_{\infty}, C_{i \varepsilon}=-\operatorname{Re} \eta \vec{u}^{(i)}(0) \nabla \vec{u}^{(i)}(\varepsilon) U_{\infty} .
\end{gathered}
$$

From Lemma 6, (49) and continuity of embeddings $W^{s+1, r}(\Omega) \mapsto C^{1}(\Omega)$ and $W^{s, r}(\Omega) \mapsto C(\Omega)$ it follows that $B_{i \varepsilon} \rightarrow B_{i}$ in $C(\Omega)$ as $\varepsilon \rightarrow 0$. In particular, continuity of embedding $C(\Omega) \mapsto L^{r}(\Omega) \mapsto \mathcal{W}^{s-1, r}(\Omega)$ implies that $B_{i \varepsilon}$ converges to $B$ in $\mathcal{W}^{s-1, r}(\Omega)$. On the other hand, due to Theorem 3 the functions $\vec{w}_{\varepsilon}^{(i)}$

$$
C_{i \varepsilon}=-\operatorname{Re} \vec{u}^{(i)}(0) \nabla \vec{u}^{(i)}(\varepsilon) U_{\infty} \rightarrow-\operatorname{Re} \vec{u}^{(i)}(0) \nabla \vec{u}^{(i)}(0) U_{\infty}=C_{i} \text { in } W^{s, r}(\Omega) .
$$

Theorem 3 provides a weak convergence of $\psi_{i \varepsilon} \rightarrow \psi_{i}$ in $\mathbb{W}^{-s, r}(\Omega)$ that results to the relationship

$$
\int_{\Omega} \psi_{i \varepsilon} C_{i \varepsilon} d x=\left\langle\psi_{i \varepsilon}, C_{i \varepsilon}\right\rangle_{0} \rightarrow\left\langle\psi_{i}, C_{i}\right\rangle_{0} \text { as } \varepsilon \rightarrow 0
$$

Finally, we note that $A \in C^{1}(\Omega) \subset W^{s, r}(\Omega)$ and $\omega_{i_{\varepsilon}} \rightarrow \omega_{i}$ weakly in $\mathbb{W}^{-s, r}(\Omega)$, which gives

$$
\begin{gathered}
\int_{\Omega} \omega_{i \varepsilon} A d x=\left\langle\omega_{i \varepsilon}, A\right\rangle_{0} \rightarrow\left\langle\omega_{i}, A\right\rangle_{0} \text { as } \varepsilon \rightarrow 0 \text {. (64) } \mathbf{N}(\varepsilon) \text { and } g(\varepsilon) \text { to (59) we obtain } \\
L_{\varepsilon, e}=O(\varepsilon)-\sum_{i=1}^{2} U_{\infty} \operatorname{Re} \int_{\Omega}\left[\rho_{i}(\varepsilon)\left(\vec{u}^{(i)}(\varepsilon) \cdot \nabla \eta\right) \mathbb{D} \vec{u}^{(i)}(\varepsilon)\right] d x- \\
-\sum_{i=1}^{2} U_{\infty} \int \sum_{\Omega}\left(\sum_{j=1}^{2} \mu_{i j}\left(\mathbb{D}^{\mathbb{T}} \nabla\left(\vec{u}^{(j)}(\varepsilon)\right)-\nabla\left(\mathbb{D} \vec{u}^{(j)}(\varepsilon)\right)+\left(\mathbb{D}^{\mathbb{T}} \nabla\left(\vec{u}^{(j)}(\varepsilon)\right)-\nabla\left(\mathbb{D} \vec{u}^{(j)}(\varepsilon)\right)\right)^{T}\right)-q_{i}(\varepsilon) \mathbb{D}^{\mathbb{T}}\right) \nabla \eta d x+ \\
+\sum_{i=1}^{2} U_{\infty} \int_{\Omega}\left(\sum_{j=1}^{2} \mu_{i j}\left(\nabla\left(\vec{u}^{(j)}(\varepsilon)\right)+\nabla\left(\vec{u}^{(j)}(\varepsilon)\right)^{T}-\operatorname{div}\left(\vec{u}^{(j)}(\varepsilon)\right) \mathbf{I}\right)\right)(D \vec{T})^{T} \nabla d x
\end{gathered}
$$

Combining (62) - (64) we obtain

$$
L_{\varepsilon, u}(\vec{T}) \rightarrow L_{u}(\vec{T}) \text { as } \varepsilon \rightarrow 0 .
$$

To pass to the limit in the expression for $L_{\varepsilon, e}$, we note that $\vec{u}^{(i)}(\varepsilon)$ are bounded in $C^{1}(\Omega)$, and $\rho_{i}(\varepsilon), q_{i}(\varepsilon)$ are bounded in $C(\Omega)$. Substituting representation (44) for
As noted above $\vec{u}^{(i)}(\varepsilon) \rightarrow \vec{u}^{(i)}(0)$ in $C^{1}(\Omega)$ and $\left(q_{i}(\varepsilon), \rho_{i}(\varepsilon)\right) \rightarrow\left(q_{i}(0), \rho_{i}(0)\right)$ in $C(\Omega)$. Thus we obtain

$$
L_{\varepsilon, e}(\vec{T}) \rightarrow L_{e}(\vec{T}) \text { with } \varepsilon \rightarrow 0
$$

Letting $\varepsilon \rightarrow 0$ into (57) and using relationships (65), (66) we arrive to (54). Theorem is proved.

Formulas similar to (53) are widely used to solve shape optimization problems. Standard gradient scheme in the shape optimization is as follows. Let us choose an arbitrary compact set $S \subset B$ as the starting point of the iteration process. Then we find the vector field $\vec{T}$ such that $d J(\Omega)[\vec{T}]<0$. A set $S_{\varepsilon}=(\mathbf{I}+\varepsilon \vec{T})(S)$ can be accepted as the next iteration step. Then we take $\Omega=\Omega(\varepsilon)$ and repeat the calculation. It is hoped that after a certain number of steps we will obtain a shape close to the optimum. Realization of such a scheme requires an efficient method for calculation of the firstorder configuration derivative $d J$. However, from the standpoint of numerical methods, representation (53) can not be a good basis for the calculation.

Further we designate the problem (6) with $\mathbf{N}=\mathbf{I}$ (unperturbed case) as the state equation, and solutions to this problem $\vec{\theta}=\left(\vec{v}^{(1)}, \vec{v}^{(2)}, \pi_{1}, \pi_{2}, \varphi_{1}, \varphi_{2}\right)$, $\vec{\zeta}=\left(\zeta_{1}^{(1)}, \zeta_{2}^{(1)}, \zeta_{1}^{(2)}, \zeta_{2}^{(2)}\right)$ and $\vec{m}=\left(m_{1}, m_{2}\right)$ as the state variables. State variables completely describe the flow in the unperturbed domain $\Omega$, and do not depend on the vector field $T$. Theorem 4 shows that the derivative of functional $d J\left(\Omega_{\varepsilon}\right)$ consists of two parts: $L_{u}$ and $L_{e}$. Geometric part $L_{e}$ is simply the integral of a linear form of $T$ over $\Omega$. The coefficients of this form are defined by state variables. Hence, it is not difficult to calculate $L_{e}$ for all smooth $T$. The dynamic part $L_{u}$, in contrast, depends on $T$ implicitly. To calculate $L_{u}$, it is necessary to find a very weak solution of the linearized problem, and then to apply this solution to (6). A very weak solution is given by Theorem 3 and depends on $T$ implicitly. If you need to find $L_{u}$ for another 
vector field $\vec{T}$, you have to repeat all calculations. Fortunately, the situation can be radically improved if $L_{u}$ will be expressed in terms of so-called adjoint state.

A known fact of the optimization theory is that in order to calculate the first- order configuration derivative $d J$ of functional, it is not required to calculate the material or shape derivatives of solutions to the boundary value problem. In addition, $d J$ can be expressed as an integral of a linear form of $\vec{T}$ with coefficients depending only on the state variables and the adjoint state, which is defined as follows.

The adjoint state is the solution $\vec{h}_{*}=\left(\vec{h}_{*}^{(1)}, \vec{h}_{*}^{(2)}\right), \vec{h}_{*}^{(i)}=\left(\vec{w}_{*}^{(i)} ; \omega_{i}^{*}, \psi_{i}^{*}, \xi_{1}^{(i)^{*}}, \xi_{2}^{(i)^{*}} ; n_{i}^{*}\right) \in W^{s+1, r}(\Omega) \times W^{s, r}(\Omega) \times \mathbb{R}$, to the conjugate problem

$$
\begin{gathered}
\sum_{j=1}^{2} \mu_{j i}\left(\Delta \vec{w}_{*}^{(j)}-\nabla \omega_{j}^{*}\right)-\operatorname{Re} \mathcal{H}_{i}^{*}\left(\vec{w}_{*}^{(j)}\right)+(-1)^{i+1} a\left(\vec{w}_{*}^{(2)}-\vec{w}_{*}^{(1)}\right)+\psi_{i}^{*} \cdot \nabla \varphi_{i}+\sum_{j=1}^{2} \zeta_{j}^{(i)} \cdot \nabla \xi_{j}^{(i)^{*}}=\vec{B}^{(i)} \text { in } \Omega, \\
\operatorname{div} \vec{w}_{*}^{(i)}=\Pi \sum_{k=1}^{2}\left[\hat{\beta}_{k i}^{*} \psi_{k}^{*}+\sum_{j=1}^{2}\left(n_{k}^{*} \chi_{k j}^{0} \widetilde{\beta}_{j i}^{*}+\mu_{k j} \beta_{j i}^{*} \omega_{k}^{*}\right)\right]+\Pi A \text { in } \Omega, \\
-\operatorname{div}\left(\psi_{i}^{*} \vec{u}^{(i)}\right)+\tau_{i i} \psi_{i}^{*}=\operatorname{Re} \mathcal{M}_{i}^{*}\left(\vec{w}_{*}^{(j)}\right)+\sum_{k=1}^{2}\left[\hat{\alpha}_{k i}^{*} \psi_{k}^{*}+\sum_{j=1}^{2}\left(n_{k}^{*} \chi_{k j}^{0} \widetilde{\alpha}_{j i}^{*}+\mu_{k j} \alpha_{j i}^{*} \omega_{k}^{*}\right)\right]+C_{i} \text { in } \Omega, \\
\vec{u}^{(i)} \nabla \xi_{j}^{(i)^{*}}+\tau \xi_{i i} \xi_{j}^{(i)^{*}}=\widetilde{\gamma}_{i j}^{*} \sum_{k=1}^{2} n_{k}^{*} \chi_{k i}^{0} \text { in } \Omega, \\
n_{i}^{*}=\iint_{\Omega}^{0}\left(\gamma_{i}^{*} \sum_{j=1}^{2} \mu_{j i} \omega_{j}^{*}+\hat{\gamma}_{i}^{*} \psi_{i}^{*}\right) d x, \\
\vec{w}_{*}^{(j)}=0 \text { on } \partial \Omega, \quad \psi_{i}^{*}=0 \text { on } \sum_{\text {out }}^{i}, \xi_{j}^{(i)^{*}}=0 \text { on } \Sigma_{i n}^{i}, \Pi \omega_{i}^{*}=\omega_{i}^{*}, i, j=1,2 .
\end{gathered}
$$

Here the coefficients and given functions are defined through the state variables as in the problem (40) and (42); $A, B_{i}, C_{i}$ are defined by the formulas (55). Therefore, the adjoint state is completely defined by the state variables, and does not depend on $\vec{T}$. Let us note that $B_{i} \in \mathcal{W}^{s-1, r}(\Omega)$ and $A, C_{i} \in W^{s, r}(\Omega)$. Consequently, according to Theorem 2 , the boundary value problem for adjoint state has a unique solution of class $W^{s+1} r(\Omega) \times W^{s, r}(\Omega) \times \mathbb{R}$. Therefore, the adjoint state is well-defined and unique. The following theorem shows that $L_{u}$ can be represented as an integral of a linear form of $\vec{T}$.

Theorem 5. 1 Under the assumptions of Theorem 4, the following formula is valid

$$
L_{u}(\vec{T})=\sum_{i=1}^{2} \int_{\Omega}\left\{\vec{w}_{*}^{(i)}\left(\mathcal{D}_{i}^{*}+(-1)^{i} \mathcal{E}^{*}\right)+d^{*}\left(\hat{\delta}_{i}^{*} \psi_{i}^{*}+\sum_{j=1}^{2}\left(\tau{ }_{j i} \xi_{j}^{(i)^{*}}+n_{i}^{*} \chi_{i j}^{0} \widetilde{\delta}_{j}^{*}+\mu_{i j} \delta_{j}^{*} \omega_{i}^{*}\right)\right)\right\} d x,
$$

where $\vec{h}_{*}=\left(\vec{h}_{*}^{(1)}, \vec{h}_{*}^{(2)}\right), \vec{h}_{*}^{(i)}=\left(\vec{w}_{*}^{(i)}, \omega_{i}^{*}, \psi_{i}^{*}, \xi_{1}^{(i)^{*}}, \xi_{2}^{(i)^{*}}, n_{i}^{*}\right)$ is the adjoint state (solution to the problem (67)) and

$$
\begin{gathered}
\mathcal{D}_{i}^{*}=-\operatorname{Re\rho }\left(\vec{u}^{(i)} \cdot \nabla\left(\mathbb{D} \vec{u}^{(i)}\right)+\mathbb{D}^{\mathbb{T}} \vec{u}^{(i)} \cdot \nabla \vec{u}^{(i)}\right)+\sum_{j=1}^{2} \mu_{i j}\left(\operatorname{div}\left(\mathbb{T} \nabla \vec{u}^{(i)}\right)+\mathbb{D}^{\mathbb{T}} \Delta \vec{u}^{(i)}+\Delta\left(\mathbb{D} \vec{u}^{(i)}\right)\right), \\
\mathcal{E}^{*}=a \mathbb{T}\left(\vec{u}^{(2)}-\vec{u}^{(1)}\right) ; \quad d^{*}=\frac{1}{2} \operatorname{Tr}(\mathbb{D})=\operatorname{div} \vec{T} \cdot \mathbf{I}, \quad \mathbb{D}=\operatorname{div} \vec{T} \cdot \mathbf{I}-D \vec{T}, \mathbb{T}=\operatorname{div} \vec{T} \mathbf{I}-D \vec{T}-(D \vec{T})^{T} .
\end{gathered}
$$

Proof. Choosing $\left(\vec{H}^{(i)}, G_{i}, F_{i}, M_{1 i}, M_{2 i}, s_{i}\right)=\left(\vec{B}^{(i)}, A, C_{i}, 0,0,0\right), i=1,2$, and applying Theorem 3, we obtain the formula

$$
\begin{gathered}
L_{u}(\vec{T})=\sum_{i=1}^{2}\left\{\left\langle\overrightarrow{\mathrm{B}}^{(i)}, \vec{w}^{(i)}\right\rangle_{1}+\left\langle\omega_{i}, A\right\rangle_{0}+\left\langle\psi_{i}, C_{i}\right\rangle_{0}\right\}=\sum_{i=1}^{2} \int_{\Omega}\left\{\vec{w}_{*}^{(i)}\left(\mathcal{D}_{i}^{*}+(-1)^{i} \mathcal{E}^{*}\right)+\right. \\
\left.+d^{*}\left(\hat{\delta}_{i}^{*} \psi_{i}^{*}+\sum_{j=1}^{2}\left(\tau{ }_{j i} \xi_{j}^{(i)^{*}}+n_{i}^{*} \chi_{i j}^{0} \tilde{\delta}_{j}^{*}+\mu_{i j} \delta_{j}^{*} \omega_{i}^{*}\right)\right]\right\} d x .
\end{gathered}
$$

Thus we shave studied properties of the boundary value problem (6) solutions depending on the shape of the obstacle in a flow of fluid, which allowed the study of the properties of this problem solution functional. Formulas for shape derivative of this functional were obtained, which can be the basis of building and implementation of numerical calculations to find the optimal shape of the obstacle in a flow of mixture of viscous compressible fluids. 


\section{REFERENCES}

1. Novo S. Compressible Navier-Stokes model with inflow-outflow boundary conditions. J. Math. Fluid Mech., 2005, vol. 7, pp. 485-514.

2. Girinon V. Navier-Stokes equations with nonhomogeneous boundary conditions in a bounded three-dimensional domain. $J$. Math. Fluid Mech., 2011, vol. 13, pp. 309-339.

3. Farwig R. Stationary solutions of compressible Navier-Stokes equations with slip boundary condition. Comm. Partial Dierential Equations, 1989, vol. 14, pp. 1579-1606.

4. Kweon J. R., Kellogg R. B. Compressible Navier-Stokes equations in a bounded domain with inflow boundary condition. SIAM J. Math. Anal., 1997, vol. 28, pp. 94-108.

5. Kweon J. R., Kellogg R. B. Regularity of solutions to the Navier-Stokes equations for compressible barotropic flows on a polygon. Arch. Ration. Mech. Anal. 2000, vol. 163, pp. 35-64.

6. Plotnikov P.I., Sokolovski Zh. Statsionarnye kraevye zadachi dlya uravneniy Nav'e-Stoksa s pokazatelem adiabaty [Boundary Value Problems for Navier-Stokes Equations with Adiabatic Exponent $\gamma<3 / 2$ ]. Doklady Akademii Nauk [Proc. of the RAS], 2004, vol. 397, no. 2, pp. 166-169.

7. Plotnikov P., Sokolowski J.Compressible Navier-Stokes equations: Theory and shape optimization. Basel: Springer, 2012.474 p.

8. Plotnikov P. I., Ruban E. V., Sokolowski J. Inhomogeneous boundary value problems for compressible Navier-Stokes and transport equations.J. Math. Pures Appl., 2009, vol. 92, pp. 113-162.

9. Zhalnina A.A., Kucher N.A. On the Well-Posedness of an Inhomogeneous Boundary Value Problem for the Equations of Mixtures of Viscous Compressible Fluids. Journal of Applied and Industrial Mathematics, 2015, vol. 9, no. 4, pp. 598-610. doi: 10.1134/ S199047891504016X

10. Sokolowski J., Zolesio J.-P. Intro duction to Shape Optimization. Shape Sensitivity Analysis. Springer Ser. Comput. Math. 16. Berlin: Springer, 1992. $250 \mathrm{p}$.

11. Bucur D., Buttazzo G. Variational Methods in Shape Optimization Problems. Boston: Birkhauser, 2005. 216 p.

12. Delfour M.C., Zolesio J.-P.Shapes and Geometries, Adv. Design Control 22. Philadelphia: SIAM, 2011.501 p.

13. Henrot A., Pierre M. Variation et optimisation de formes: une analyse geometrique. Paris: Springer, 2005. 334 p.

14. Kawohl B., Pironneau O., Tartar L., Zolesio J.-P.Optimal Shape Design, Lecture Notes in Math.Berlin: Springer, 2000. 388 p.

15. Novotny A., Sokolowski J. Topological Derivatives in Shape Optimization. Series: Interaction of Mechanics and Mathematics. Berlin: Springer, 2013. 412 p.

16. Feireisl E. Shape optimization in viscous compressible fluids. Appl. Math. Optim., 2003, vol. 47, pp. 59-78.

17. Plotnikov P., Sokolowski J. Shape derivative of drag functional.SIAM J.Control Optim., 2010, vol. 48, pp. 4680-4706.

18. Plotnikov P.I., Ruban E.V., Sokolowski J. Inhomogeneous boundary value problems for compressible Navier-Stokes equations, well-posedness and sensitivity analysis. SIAM J. Math. Anal., 2008, vol. 40, pp. 1152-1200

19. Plotnikov P., Sokolowski J. Domain dependence of solutions to compressible Navier-Stokes equations. SIAM J. Control Optim, 2006, vol. 45, pp. 1165-1197.

20. Plotnikov P., Sokolowski J. On compactness, domain dependence and existence of steady state solutions to compressible isothermal Navier-Stokes equations.J. Math. Fluid Mech., 2005, vol. 7, pp. 529-573.

21. Rajagopal K. R., Tao L.Mechanics of mixtures. Singapore: World Sci., 1995. 195 p.

22. Krayko A.N., Nigmatulin R.I. Mekhanika mnogofaznykh sred [Mechanics of multiphase media]. Itogi nauki $i$ tehniki. Seriya: gidromekhanika [Results of science and technology. Series: Hydromechanics], 1972, vol. 6, pp. 93-174.

23. Nigmatulin R.I.Dinamika mnogofaznykh sred. Chast' 1 [Dynamics of multiphase media] Part 1. Moscow: Nauka Publ., 1987. 464 p.

24. Sobolev S. L. Nekotorye primeneniya funktsional'nogo analiza v matematicheskoy fizike [Some applications of functional analysis in mathematical physics]. Moscow: Nauka Publ., 1988. 333 p.

25 Berg Y., Lefstrem Y. Interpolyatsionnye prostranstva. Vvedenie [Interpolation spaces. Introduction]. Moscow: Mir Publ., 1980. 264 p.

26. Galdi G. An introduction to the mathematical theory of the Navier-Stokes equations: Steady-State Problems. Springer Monographs in Mathematics. New-York: Springer Science, 2011. 1018 p.

Nikolay A. Kucher

Dr.Sci.(Phys.-Math.), Professor of fundamental mathematics, Kemerovo State University, Kemerovo, Russian Federation

Aleksandra A. Zhalnina

Senior lecturer in fundamental mathematics, Kemerovo State University, Kemerovo, Russian Federation 